\title{
Nictaba Homologs from Arabidopsis thaliana Are Involved in Plant Stress Responses
}

\author{
Lore Eggermont, Karolina Stefanowicz ${ }^{\dagger}$ and Els J. M. Van Damme* \\ Laboratory of Biochemistry and Glycobiology, Department of Molecular Biotechnology, Faculty of Bioscience Engineering, \\ Ghent University, Ghent, Belgium
}

OPEN ACCESS

Edited by:

Maoteng Li,

Huazhong University of Science and Technology, China

Reviewed by: Yan Long,

Chinese Academy of Agricultural

Sciences, China

Chunhua Fu,

Huazhong University of Science and Technology, China

*Correspondence:

Els J. M. Van Damme elsjm.vandamme@ugent.be

tPresent address: Karolina Stefanowicz,

Plant Systems Biology Team, Department of Integrative Plant Biology, Institute of Plant Genetics,

Polish Academy of Sciences, Poznan, Poland

Specialty section:

This article was submitted to Plant Breeding,

a section of the journal

Frontiers in Plant Science

Received: 15 July 2017 Accepted: 18 December 2017 Published: 10 January 2018

Citation:

Eggermont L, Stefanowicz K and Van Damme EJM (2018) Nictaba Homologs from Arabidopsis thaliana Are Involved in Plant Stress Responses. Front. Plant Sci. 8:2218.

doi: 10.3389/fp/s.2017.02218
Plants are constantly exposed to a wide range of environmental stresses, but evolved complicated adaptive and defense mechanisms which allow them to survive in unfavorable conditions. These mechanisms protect and defend plants by using different immune receptors located either at the cell surface or in the cytoplasmic compartment. Lectins or carbohydrate-binding proteins are widespread in the plant kingdom and constitute an important part of these immune receptors. In the past years, lectin research has focused on the stress-inducible lectins. The Nicotiana tabacum agglutinin, abbreviated as Nictaba, served as a model for one family of stress-related lectins. Here we focus on three non-chimeric Nictaba homologs from Arabidopsis thaliana, referred to as AN3, AN4, and AN5. Confocal microscopy of ArathNictaba enhanced green fluorescent protein (EGFP) fusion constructs transiently expressed in N. benthamiana or stably expressed in A. thaliana yielded fluorescence for AN4 and AN5 in the nucleus and the cytoplasm of the plant cell, while fluorescence for AN3 was only detected in the cytoplasm. RT-qPCR analysis revealed low expression for all three ArathNictabas in different tissues throughout plant development. Stress application altered the expression levels, but all three ArathNictabas showed a different expression pattern. Pseudomonas syringae infection experiments with AN4 and AN5 overexpression lines demonstrated a significantly higher tolerance of several transgenic lines to $P$. syringae compared to wild type plants. Finally, AN4 was shown to interact with two enzymes involved in plant defense, namely TGG1 and BGLU23. Taken together, our data suggest that the ArathNictabas represent stress-regulated proteins with a possible role in plant stress responses. On the long term this research can contribute to the development of more stress-resistant plants.

Keywords: plant lectin, Nictaba homolog, Arabidopsis thaliana, ArathNictaba, abiotic stress, biotic stress, interaction partner, plant defense

Abbreviations: AA, amino acids; ABA, abscisic acid; AN3, ArathNictaba3; AN4, ArathNictaba4; AN4-HIS, C-terminally His6-tagged AN4; AN5, ArathNictaba5; ArathNictabas, Nictaba homologs from A. thaliana; CTAB, hexadecyltrimethylammonium bromide; EGFP, enhanced green fluorescent protein; GlcNAc, $N$-acetyl-D-glucosamine; IPTG, isopropyl $\beta$-D-1-thiogalactopyranoside; IZ, imidazole; MeJA, methyl jasmonate; MS, Murashige and Skoog; NLS, nuclear localization signal; PAGE, polyacrylamide gel electrophoresis; PB, phosphate buffer; PCR, polymerase chain reaction; PP2A, protein phosphatase 2A; qPCR, quantitative PCR; RT, reverse transcriptase; SA, salicylic acid; SDS, sodium dodecyl sulfate; T-DNA, transferred DNA; TBS, Tris-buffered saline; WT, wild type. 


\section{INTRODUCTION}

Plants are constantly exposed to multiple abiotic and biotic stresses. The innate immune system of plants encompasses different immune receptors located either at the cell surface or in the cytoplasmic compartment and allows plants to counteract pathogen attack and survive unfavorable conditions. Evidence has been presented that plant lectins play an important role in the plant innate immune system as immune receptors and/or defense proteins (Peumans and Van Damme, 1995; Lannoo and Van Damme, 2010, 2014). Lectins or carbohydrate-binding proteins are widespread in the plant kingdom and can be classified in 12 lectin families (Van Damme et al., 2008). Representative proteins for six out of these lectin families are known as stress-inducible, nucleocytoplasmic lectins. These proteins are low abundant or may even be absent under normal growth conditions, but their expression is elevated when the plant is exposed to stress (Lannoo and Van Damme, 2010).

The Nictaba family represents a family of nucleocytoplasmic lectins and is known to be widespread in the plant kingdom (Delporte et al., 2015; Van Holle et al., 2017). The family was named after the Nicotiana tabacum agglutinin (abbreviated as Nictaba), the first lectin of this family (Chen et al., 2002). Nictaba consists of two identical non-covalently linked subunits of $19 \mathrm{kDa}$ (Chen et al., 2002). Hapten inhibition assays revealed the specific interaction of Nictaba with GlcNAc oligomers. In addition, glycan array analyses showed interaction of Nictaba with the core $\mathrm{GlcNAc}_{2} \mathrm{Man}_{3}$ of high-mannose and complex $\mathrm{N}$-glycans (Lannoo et al., 2006). Based on sequence alignments and molecular modeling studies Schouppe et al. (2010) predicted that Trp15, Trp22, Glu138, and Glu145 are conserved AA residues in the carbohydrate binding site. Mutational analysis revealed that the tryptophan residues play an important role in the carbohydrate binding activity of Nictaba (Schouppe et al., 2010). Transcript levels for Nictaba were not detectable under normal plant growth conditions but increased several fold after plant exposure to stress situations such as jasmonate treatment and insect herbivory (Chen et al., 2002; Vandenborre et al., 2009). Immunocytochemical localization studies first showed that Nictaba locates to the nucleus and the cytoplasm of leaf parenchyma cells (Chen et al., 2002). Lannoo et al. (2006) confirmed the nucleocytoplasmic localization of Nictaba using fusion constructs with EGFP transiently and stably transformed in different plant systems. Furthermore, using tobacco plants stably expressing a Nictaba promoter- $\beta$-glucuronidase fusion construct, Delporte et al. (2013) showed promoter activity in the cotyledons, the leaves as well as the roots of young plants, but promoter activity decreased when the plants grow older. Taking into account the carbohydrate dependent interaction of Nictaba with O-GlcNAc modified core histones, it was suggested that Nictaba might fulfill a role in the remodeling of chromatin conformation, and as such, changing of gene expression in response to stress (Schouppe et al., 2011; Delporte et al., 2014).

The genome of Arabidopsis thaliana contains 30 sequences with a Nictaba domain. A large number of these Nictaba homologs possess an N-terminal F-box domain, next to the carbohydrate binding domain. In addition, four Nictaba homologs were identified with an N-terminal Toll/Interleukin1 receptor domain and one sequence contains an $\mathrm{N}$-terminal avirulence induced gene 1-type G domain (Eggermont et al., 2017). This study will focus on the ArathNictabas containing only a Nictaba domain in order to elucidate the biological importance of this Nictaba domain. Based on protein sequence alignments with Nictaba, the length of the $\mathrm{N}$-terminal domains and identified ESTs three ArathNictabas, in particular AN3, AN4, and AN5, were selected. The localization in the cell, the expression in different plant tissues under normal growth or stress conditions and the interaction partners have been investigated to get insight into the biological importance of these proteins in the stress responses of $A$. thaliana.

\section{MATERIALS AND METHODS}

\section{Plant Material and Growth Conditions}

Wild type (WT) A. thaliana seeds, ecotype Col-0, were purchased from Lehle Seeds (Round Rock, TX, United States). Arabidopsis seeds were grown in pots containing commercial soil or in artificial soil (Jiffy-7, $44 \mathrm{~mm} \varnothing$, distributed by InterGrow, Aalter) in a growth chamber at $21^{\circ} \mathrm{C}$ with a $16 / 8 \mathrm{~h} \mathrm{light/dark} \mathrm{photoperiod}$ after a 3 days stratification period at $4^{\circ} \mathrm{C}$ in the dark. The light intensity in the controlled growth chamber was approximately $100 \mu \mathrm{mol} / \mathrm{m}^{2} . \mathrm{s}$ [Radium Spectralux plus white (58W) lamps]. Alternatively, seeds were grown in vitro, therefore seeds were surface sterilized in $70 \%$ ethanol for $2 \mathrm{~min}$ followed by $5 \%$ bleach for $10 \mathrm{~min}$. Afterward seeds were rinsed with sterile distilled water. The sterilized Arabidopsis seeds were sown in vitro on solid MS medium (4.3 g/L MS salts with vitamins and nutrients (Duchefa), $30 \mathrm{~g} / \mathrm{L}$ sucrose [Applichem), $\mathrm{pH}$ 5.7-5.8 (adjusted with $0.5 \mathrm{M} \mathrm{NaOH}$ ) and $8 \mathrm{~g} / \mathrm{L}$ plant agar (Duchefa)]. After a 3 days stratification period at $4^{\circ} \mathrm{C}$ in the dark, the plates were transferred to a growth chamber at $21^{\circ} \mathrm{C}$ with a $16 / 8 \mathrm{~h}$ light/dark photoperiod.

Wild type $N$. benthamiana seeds were supplied by Dr. Verne A. Sisson (Oxford Tobacco Research Station, Oxford, NC, United States). For transient transformation, the tobacco seeds were sown in pots containing commercial soil and cultivated in a growth chamber at $25^{\circ} \mathrm{C}$ with a $16 / 8 \mathrm{~h}$ light/dark photoperiod.

\section{Cloning of the ArathNictaba Sequences}

Plant samples were homogenized using a mortar and a pestle, and total RNA was extracted using TRI Reagent ${ }^{\circledR}$ according to the instructions of the manufacturer (Sigma-Aldrich). The RNA samples were treated with DNase I (Life Technologies) according to the manufacturer's instructions. The RNA concentration was measured with a Nanodrop 2000 spectrophotometer (Thermo Scientific). cDNA was synthesized from $1 \mu \mathrm{g}$ of total RNA using the moloney murine leukemia virus $\mathrm{RT}$ and oligo $(\mathrm{dT})_{25}$ primer (Life Technologies).

The full length cDNA sequences encoding AN3, AN4, and AN5 were retrieved by RT-PCR reactions with gene specific primers (Supplementary Table 1). The PCR reaction mixture was as follows: $2 \mu \mathrm{l}$ cDNA, $2 \mu \mathrm{l} 10 \mathrm{mM}$ dNTPs (Thermo Fisher Scientific), $2.5 \mu \mathrm{l} 10 \times \mathrm{RxN}$ buffer (VWR), $1 \mu \mathrm{l} 5 \mu \mathrm{M}$ 
forward and $1 \mu \mathrm{l} 5 \mu \mathrm{M}$ reverse primer (Life Technologies), $0.75 \mu \mathrm{l} 50 \mathrm{mM} \mathrm{MgCl}_{2}, 0.125 \mu \mathrm{l}$ Platinum ${ }^{\circledR}$ Pfx DNA Polymerase (Life Technologies) and water up to the volume of $25 \mu \mathrm{l}$. The PCR conditions used were: $2 \min 95^{\circ} \mathrm{C}-30-35 \times(15 \mathrm{~s}$ $\left.94^{\circ} \mathrm{C}-30 \mathrm{~s} 47-50^{\circ} \mathrm{C}-1 \min 72^{\circ} \mathrm{C}\right)-5 \mathrm{~s} 72^{\circ} \mathrm{C}$. After cloning these sequences in the pJET2.1 vector with the CloneJET PCR Cloning kit (Life Technologies), the constructs were checked by agarose gel electrophoresis and sequenced (LGC Genomics, Berlin, Germany) to confirm the correct cDNA sequence.

cDNA quality was checked by RT-PCR using primers specific for the PP2A gene (Supplementary Table 2). The PCR reaction mixture was the same as previously mentioned except for the buffer $(10 \times$ EXTRA buffer, VWR) and the enzyme (Taq DNA polymerase, VWR). The PCR conditions were as follows: $5 \mathrm{~min}$ $95^{\circ} \mathrm{C}-45 \times\left(45 \mathrm{~s} 94^{\circ} \mathrm{C}-45 \mathrm{~s} 55^{\circ} \mathrm{C}-30 \mathrm{~s} 72^{\circ} \mathrm{C}\right)-5 \mathrm{~min}$ $72^{\circ} \mathrm{C}$. PCR amplification products were checked by agarose gel electrophoresis.

\section{Construction of EGFP Fusion Constructs}

Coding sequences for the ArathNictabas were $\mathrm{N}$ - and C-terminally fused to EGFP using the Gateway ${ }^{\circledR}$ Cloning Technology (Life Technologies, Carlsbad, CA, United States). The cloned full length cDNA sequences (in the pJET1.2 vector) were used as a template to amplify the open reading frames with primers to attach attB sites. In the first PCR, the first part of the attB site is attached using Platinum ${ }^{\circledR} P f x$ DNA Polymerase (Life Technologies) and primers with a gene specific part (with or without stop codon) and the first part of the attB site (Supplementary Table 3). In the second PCR, a 1:5 dilution of the first PCR product was used as a template in combination with primers to complete the att $\mathrm{B}$ sites (Supplementary Table 3 ). The PCR conditions for the first PCR were as follows: $2 \min 94^{\circ} \mathrm{C}-30 \times\left(15 \mathrm{~s} 94^{\circ} \mathrm{C}-30 \mathrm{~s}\right.$ $\left.50^{\circ} \mathrm{C}-1 \min 72^{\circ} \mathrm{C}\right)-5 \min 72^{\circ} \mathrm{C}$. The conditions for the second PCR were: $2 \min 94^{\circ} \mathrm{C}-5 \times\left(15 \mathrm{~s} 94^{\circ} \mathrm{C}-30 \mathrm{~s} 48^{\circ} \mathrm{C}-1 \mathrm{~min}\right.$ $\left.72^{\circ} \mathrm{C}\right)-25 \times\left(15 \mathrm{~s} 94^{\circ} \mathrm{C}-30 \mathrm{~s} 55^{\circ} \mathrm{C}-1 \min 72^{\circ} \mathrm{C}\right)-5 \min 72^{\circ} \mathrm{C}$. After checking the PCR products by agarose gel electrophoresis, the PCR fragments were used in a BP recombination reaction with the pDONR221 vector. The att B PCR products and the pDONR221 vector were incubated overnight in equimolar amounts with the BP Clonase ${ }^{\circledR}$ II enzyme mix. The next day, the resulting entry clones were transformed into heat-shock competent Escherichia coli cells (TOP10) and transformants were selected on LB agar plates with $50 \mu \mathrm{g} / \mathrm{mL}$ kanamycin. Subsequently, transformants were checked with colony PCR and agarose gel electrophoresis. The entry clones were extracted using the GeneJET Plasmid Miniprep kit (Thermo Fisher Scientific) according to the manufacturer's instructions. After sequencing (LGC Genomics, Berlin, Germany) the entry clones were used in a LR recombination reaction with the destination vectors pK7WGF2,0 or pK7FWG2,0 to make the N-terminal and C-terminal fusion constructs with EGFP, respectively (Karimi et al., 2002). This recombination reaction was incubated overnight according to the Gateway ${ }^{\circledR}$ manual and expression clones were transformed into $E$. coli TOP10 cells using heat shock. Transformants were selected on LB agar plates with
$75 \mu \mathrm{g} / \mathrm{mL}$ spectinomycin and screened with colony PCR using gene specific and EGFP primers (Supplementary Table 3).

The expression vectors containing the different EGFP fusion constructs were introduced into Agrobacterium tumefaciens C58C1 pMP90 cells using triparental mating. Briefly, a donor strain (E. coli containing the expression vectors), a helper strain and the Agrobacterium were mixed together on solid YEB medium ( $5 \mathrm{~g} / \mathrm{L}$ beef extract, $5 \mathrm{~g} / \mathrm{L}$ peptone, $1 \mathrm{~g} / \mathrm{L}$ yeast extract, $5 \mathrm{~g} / \mathrm{L}$ sucrose, and $15 \mathrm{~g} / \mathrm{L}$ bacterial agar) containing $2 \mathrm{mM} \mathrm{MgSO}_{4}$. After incubation, dilution series were made and transformants were selected on YEB medium containing $75 \mu \mathrm{g} / \mathrm{mL}$ spectinomycin and $20 \mu \mathrm{g} / \mathrm{mL}$ gentamicin. After purification of the expression clones from the Agrobacterium, screening of the clones was done by PCR (Supplementary Table 3).

\section{Construction of ArathNictaba Overexpression Constructs}

The entry clones containing the AN4 and AN5 coding sequences with stop codon (see section "Construction of EGFP Fusion Constructs") were used in an LR reaction to generate the overexpression constructs. The destination vector pK7WG2,0 (Karimi et al., 2002) containing the 35 S promoter was combined with the entry clones and the Gateway LR Clonase II to get the desired expression clones (35S::AN4 and 35S::AN5). These expression clones were heat shock transformed in TOP10 E. coli cells and transformants were selected on LB agar plates containing $75 \mu \mathrm{g} / \mathrm{mL}$ spectinomycin. Transformants were screened with colony PCR using a forward primer in the $35 \mathrm{~S}$ promoter and a reverse primer in the $35 \mathrm{~S}$ terminator sequence (Supplementary Table 4). Electrocompetent A. tumefaciens GV3101 cells were transformed with these expression clones (300 ng) using electroporation with the following parameters: $2.0 \mathrm{kV}, 25 \mu \mathrm{F}$, and $200 \Omega$. Immediately after the pulse, YEB medium was added and the cells were grown on a shaker $(200 \mathrm{rpm})$ at $28^{\circ} \mathrm{C}$ for $2 \mathrm{~h}$. Transformants were selected on YEB agar plates with $75 \mu \mathrm{g} / \mathrm{mL}$ spectinomycin and screened with colony PCR using primers located in the $35 \mathrm{~S}$ promoter and terminator sequences (Supplementary Table 4).

\section{Transient Transformation of $N$. benthamiana Leaves}

Transient expression of the EGFP fusion constructs was obtained by infiltration of the transformed Agrobacterium in leaves of 4- to 6-week-old N. benthamiana plants as described by Sparkes et al. (2006). First, the Agrobacterium strains were grown in liquid YEB medium containing $75 \mu \mathrm{g} / \mathrm{mL}$ spectinomycin and $20 \mu \mathrm{g} / \mathrm{mL}$ gentamicin for 2 days at $25^{\circ} \mathrm{C}$ on a rotary shaker (200 rpm). Agrobacterium cells were harvested by centrifugation and resuspended in infiltration medium $(50 \mathrm{mM}$ MES, $2 \mathrm{mM} \mathrm{Na}_{2} \mathrm{HPO}_{4}, 0.5 \%$ glucose, $\mathrm{pH}$ 5.6). Centrifugation and resuspension were repeated twice, the second time using infiltration medium with $100 \mu \mathrm{M}$ acetosyringone. After washing, the cells were diluted to a final optical density at $600 \mathrm{~nm}$ of 0.01 , $0.05,0.1$, and 0.2 and infiltrated in the leaf epidermal cells. Two or three days post-infiltration, microscopic analysis was performed. 


\section{Stable Transformation of $A$. thaliana Plants}

Stably transformed Arabidopsis plants were created using the floral dip transformation method (Clough and Bent, 1998). Transformed seeds were selected on MS medium containing $75 \mu \mathrm{g} / \mathrm{mL}$ kanamycin (Duchefa) using the fast selection protocol according to Harrison et al. (2006). Green plantlets were transferred to new selective MS medium and afterward to artificial soil. For the ArathNictaba EGFP overexpression lines integration of the T-DNA was checked by PCR on gDNA using gene specific and EGFP primers (Supplementary Table 3). T2 generation Arabidopsis plants were used for all analyses. For the ArathNictaba overexpression lines, selection was done until the transformed plants were homozygous for the T-DNA integration (T3 and T4). Integration of the T-DNA was checked by PCR on gDNA using kanamycin resistance gene primers (Supplementary Table 4) using the following PCR program: $10^{\prime} 94^{\circ} \mathrm{C}-45 \times\left(30^{\prime \prime}\right.$ $\left.94^{\circ} \mathrm{C}-30^{\prime \prime} 48^{\circ} \mathrm{C}-1^{\prime} 72^{\circ} \mathrm{C}\right)-5^{\prime} 72^{\circ} \mathrm{C}$. The quality of the gDNA was checked with actin primers (Supplementary Table 4) using the same PCR program. Overexpression levels of the ArathNictaba genes in 15-day-old seedlings were quantified by RT-quantitative (q)PCR. Three independent homozygous single insertion lines were selected for each construct (35S::AN4 and 35S::AN5).

\section{ArathNictaba Expression during Plant Development}

For the aerial plant tissues, WT Arabidopsis seeds were sown in vitro on MS medium (until 22 days) or in artificial soil (Jiffy-7, $44 \mathrm{~mm} \varnothing$ ). The Arabidopsis plants grown in artificial soil were watered regularly and fertilizer was added once after 25 days. Whole plantlets were collected at 6,15 , and 22 days after sowing. Rosette leaves from at least five plants were harvested and pooled after 31 days. After 39 and 54 days, rosette leaves, cauline leaves, stems and flowers from at least five plants were sampled and pooled. For the root samples, WT Arabidopsis seeds were sown in expanded clay granules $(\varnothing<4 \mathrm{~mm})$. The plants were watered regularly and fertilizer was added once per week. Root samples from at least 20 plants were collected and pooled after 34,46 , and 59 days. All samples were immediately frozen in liquid nitrogen and stored at $-80^{\circ} \mathrm{C}$ prior to RNA extraction. Two biological replicates were performed and analyzed, each with two technical replicates.

\section{Hormone and Abiotic Stress Treatments}

Sixteen-day-old Arabidopsis seedlings grown in vitro on a filter paper on top of MS medium were used for the treatment with the following solutions: $100 \mu \mathrm{M}$ MeJA, $100 \mu \mathrm{M} \mathrm{ABA}, 300 \mu \mathrm{M} \mathrm{SA}$, and $150 \mathrm{mM} \mathrm{NaCl}$. Prior to use, stock solutions of the hormones (MeJA, ABA, and SA) were made in $100 \%$ ethanol and water was used in case of the salt solution. Control plants were kept on liquid MS medium containing an equal concentration of the corresponding solvent (ethanol or water). For each treatment, the filter papers with the germinated seedlings were transferred to Petri dishes filled with liquid MS medium containing either the hormone or the salt solution, and incubated at $21^{\circ} \mathrm{C}$. Heat stress was applied by incubating the plates with seedlings in the dark at $37^{\circ} \mathrm{C}$, controls were incubated at $21^{\circ} \mathrm{C}$ in the dark. For every treatment, 50 seedlings were collected at several time points $(1,3$, 5,10 , and $24 \mathrm{~h}$ ) after stress initiation. Samples were immediately frozen in liquid nitrogen and stored at $-80^{\circ} \mathrm{C}$ prior to use. Four independent biological replicates were performed for MeJA, SA and $\mathrm{NaCl}$ stress, two biological replicates were performed for $\mathrm{ABA}$ and heat stress.

\section{Biotic Stress Treatments}

Pseudomonas syringae and Botrytis cinerea infection experiments were performed with 5-week-old WT Arabidopsis plants of the Col-0 ecotype grown in artificial soil (Jiffy-7). The P. syringae pv. tomato DC3000 strain and the B. cinerea B05.10 strain were supplied by Prof. Dr. M. Höfte of the Phytopathology Lab (Ghent University, Belgium). Infection assays were performed according to Pieterse et al. (1996), Audenaert et al. (2002), and Katagiri et al. (2002), with minor modifications.

Pseudomonas syringae pv. tomato DC3000 was grown in liquid King's B medium ( $20 \mathrm{~g} / \mathrm{L}$ peptone, $1 \%$ glycerol, $1.5 \mathrm{~g} / \mathrm{L}$ $\left.\mathrm{KH}_{2} \mathrm{PO}_{4}, 1.5 \mathrm{~g} / \mathrm{L} \mathrm{MgSO}_{4} \cdot 7 \mathrm{H}_{2} \mathrm{O}, \mathrm{pH} 7.2\right)$ at $28^{\circ} \mathrm{C}$ on a rotary shaker $(200 \mathrm{rpm})$ until the culture reached the mid to late $\log$ growth phase $\left(\mathrm{OD}_{600}=0.6-1.0\right)$. After centrifugation of the culture (10 $\mathrm{min}, 2500 \mathrm{~g})$, bacterial cells were resuspended in $10 \mathrm{mM} \mathrm{MgSO}_{4}$ to obtain a solution of bacteria with an $\mathrm{OD}_{600}$ of 0.05 (corresponding to $2.5 \times 10^{7} \mathrm{cfu} / \mathrm{mL}$ ). Prior to use, $0.05 \%$ Silwet-77 (GE Specialty Materials, Switzerland) was added to the infection solution. The mock solution consisted of $10 \mathrm{mM} \mathrm{MgSO}_{4}$ containing $0.05 \%$ Silwet-77. The rosette leaves of the Arabidopsis plants were sprayed until run-off with either the infection or the mock solution. To increase the efficiency of the infection, the plants were maintained at $100 \%$ relative humidity 1 day before the treatment until 2 days after the start of the bacterial infection. Three biological replicates were performed and analyzed.

The Botrytis strain was kept on regular potato dextrose agar plates at $21^{\circ} \mathrm{C}$. Sporulation was stimulated by incubation for 10 days at $21^{\circ} \mathrm{C}$ under a $12 / 12 \mathrm{~h} \mathrm{UV/dark} \mathrm{light} \mathrm{regime.} \mathrm{After}$ 10 days, Botrytis spores were harvested by washing the plates with distilled water containing $0.01 \%$ Tween-20 (VWR). This suspension was filtered through a nylon membrane $(20 \mu \mathrm{m} \varnothing)$ and an inoculation suspension $\left(5 \times 10^{5}\right.$ conidia $\left./ \mathrm{mL}\right)$ was prepared in half strength potato dextrose broth medium. The mock solution consisted of the same medium without spores. Droplets $(10 \mu \mathrm{L})$ of the infection suspension or the mock solution were applied on the upper side of three rosette leaves from each plant. Plants were maintained in $100 \%$ relative humidity during the entire experiment. Two biological replicates were performed and analyzed.

During the infection assays, plants were kept in a Conviron growth chamber at $21^{\circ} \mathrm{C}$ with a $12 / 12 \mathrm{~h}$ light/dark photoperiod. Control plants were kept separately from infected plants. Rosette leaves of 8-10 randomly selected plants were sampled in liquid nitrogen at different time points post-infection and samples were stored at $-80^{\circ} \mathrm{C}$ prior to use.

Myzus persicae was kindly provided by Prof. Dr. Guy Smagghe (Agrozoology Lab, Ghent University, Belgium) and kept on 
sweet pepper plants under lab conditions (Shahidi-Noghabi et al., 2009). Aphid infestation was performed on 5-week-old Arabidopsis plants sown in round plastic pots $(\varnothing 11 \mathrm{~cm})$ with soil on which a transparent ventilated cage (Novolab) was placed. Sixty aphids were placed on the rosette leaves of each plant. Control plants were grown in cages without aphids. During the assay, plants were kept in a Conviron growth chamber at $21^{\circ} \mathrm{C}$ with a $12 / 12 \mathrm{~h}$ light/dark photoperiod. At indicated time points, two leaves of nine randomly chosen plants were sampled, frozen in liquid nitrogen and stored at $-80^{\circ} \mathrm{C}$ prior to use. Four biological replicates were performed and analyzed, each with two technical replicates.

\section{RT-qPCR Analysis}

Real time RT-qPCR analyses of the gene expression during development and after $P$. syringae infection, were performed using the Rotor-Gene 3000 (Corbet Life Science) and the Rotor Discs (Qiagen, Hilden, Germany). The program was as follows: $10 \min 95^{\circ} \mathrm{C}-45 \times\left(25 \mathrm{~s} 95^{\circ} \mathrm{C}-25 \mathrm{~s} 60^{\circ} \mathrm{C}-20 \mathrm{~s} 72^{\circ} \mathrm{C}\right)-5 \mathrm{~min}$ $72^{\circ} \mathrm{C}$ followed by generation of a melting curve (gradual increase from 72 to $95^{\circ} \mathrm{C}$ with $1^{\circ} \mathrm{C} /$ step). The Rotor Gene 6 software generated the raw output data (Cq values), these were statistically analyzed using the REST-384 software (Corbett Research). REST384 uses a pair wise fixed reallocation randomization test as a statistical model (Pfaffl et al., 2002).

RT-qPCR analyses of the other stress experiments and overexpression experiments were performed using the 96-well CFX Connect ${ }^{\mathrm{TM}}$ Real-Time PCR Detection System (Bio-Rad). The program was as follows: $10 \min 95^{\circ} \mathrm{C}-45 \times\left(15 \mathrm{~s} 95^{\circ} \mathrm{C}-\right.$ $25 \mathrm{~s} 60^{\circ} \mathrm{C}-20 \mathrm{~s} 72^{\circ} \mathrm{C}$ ) followed by generation of a melting curve (gradual increase from 65 to $95^{\circ} \mathrm{C}$ with $0.5^{\circ} \mathrm{C} / \mathrm{step}$ ). The CFX Manager 3.1 software (Bio-Rad) generated the raw output data which were statistically analyzed using the REST-384 software.

All reactions were conducted in a total volume of $20 \mu \mathrm{l}$ containing $1 \times$ SensiMix $^{\mathrm{TM}} \mathrm{SYBR}^{\circledR}$ No-ROX One-Step mix, $2 \mu \mathrm{l}$ undiluted cDNA template, $500 \mathrm{nM}$ gene specific forward and reverse primers (Supplementary Table 2). All gene specific qPCR primers were designed using Primer $3^{1}$. Specificity of the primers was tested in silico by BLAST search and amplicons were cloned and verified using agarose gel electrophoresis and sequencing (LGC Genomics, Berlin). Amplification efficiency of all primer pairs was determined in the CFX Manager 3.1 (Bio-Rad) and qBASE $^{\text {PLUS }}$ software (Hellemans et al., 2007). All expression data were normalized using three reference genes: PP2A, TIP41, and UBC9 (Czechowski et al., 2005). All melting curves were checked and reference gene stability and quality control of the samples were validated in the qBASE ${ }^{\text {PLUS }}$ software (Hellemans et al., 2007).

\section{P. syringae Infection of Arabidopsis Overexpression Lines}

Four-week-old Arabidopsis WT plants and three independent homozygous transgenic lines for each construct (35S::AN4 and $35 \mathrm{~S}:: A N 5)$ were inoculated with the infection $\left(1.6 \times 10^{7} \mathrm{cfu} / \mathrm{mL}\right)$ or mock solutions. Rosette leaves of three individual randomly

${ }^{1}$ http://biotools.umassmed.edu/bioapps/primer3_www.cgi selected plants were sampled at different time points postinfection ( $1-5 \mathrm{dpi}$ ). Two independent biological replicates were performed.

To measure leaf damage, six leaves per line per time point were scanned with a flatbed scanner at the highest resolution. The percentage of leaf damage was determined using the Image Analysis Software for Plant Disease Quantification Assess 2.0 (APS, St. Paul, MN, United States) using a self-written macro adjusted to our sampled leaves. The data were tested for normal distribution with the Shapiro-Wilkinson test. The MannWhitney $U$ test was used for not-normally distributed data, supplemented with a non-parametric equivalent of the Levene's test to check homogeneity of variances. The Bonferroni-Holm correction was used for multiple testing.

Using trypan blue staining (Sigma-Aldrich, Diegem, Belgium), plant cell death was visualized. One leaf of three plants per line for each time point was submerged in trypan blue solution $(0.02 \%)$ and boiled for $2 \mathrm{~min}$. Afterward, the leaves were incubated overnight at room temperature on a rotary shaker (50-100 rpm). Next, the trypan blue solution was replaced with a chloral hydrate solution $(100 \mathrm{~g} / 40 \mathrm{~mL}$ water $)$ to destain the leaves. The destained leaves were placed on a microscopy slide in $50 \%$ glycerol and pictures were taken with a Leica S8APO microscope (DFC400 camera) and Leica Plan APO 1.6× objective. The trypan blue staining was scored by estimating the percentage of blue staining or cell death. Leaves without trypan blue staining $(0 \%)$ were assigned a score 1 . Score 2 was assigned to leaves for which the percentage of cell death was $1-30 \%$. Leaves with $31-60 \%$ of cell death were assigned a score 3 . Score 4 was assigned to leaves for which the percentage of cell death was $61-100 \%$. Each transgenic line was statistically compared with the WT using a Mann-Whitney $U$ test supplemented with a non-parametric equivalent of the Levene's test to check homogeneity of variances. The Bonferroni-Holm correction was used for multiple testing.

To determine the $P$. syringae biomass, gDNA was first extracted from the infected and mock treated leaves using CTAB. The CTAB buffer (2\% CTAB, 0.1 M Tris- $\mathrm{HCl} \mathrm{pH} \mathrm{7.5,} 1.4 \mathrm{M}$ $\mathrm{NaCl}, 20 \mathrm{mM}$ EDTA) was added to $100 \mathrm{mg}$ of plant material and this mixture was incubated at $65^{\circ} \mathrm{C}$ for $90 \mathrm{~min}$ in a shaking heat block. After chloroform:isoamylalcohol (24:1) extraction the gDNA was precipitated with $100 \%$ isopropanol, washed with $76 \%$ ethanol/0.2 M NaOAc and 76\% ethanol/10 $\mathrm{mM} \mathrm{NH}_{4} \mathrm{OAc}$ and dissolved in water. Quantification of $P$. syringae biomass was performed with RT-qPCR using oprf primers targeting the outer membrane porin protein $\mathrm{F}$ gene of $P$. syringae (Brouwer et al., 2003) (Supplementary Table 2). ACT2 and PEX4 primers were used as reference genes for $A$. thaliana (Supplementary Table 2). The REST-384 software was used to calculate the ratio of P. syringae gDNA to A. thaliana gDNA (Pfaffl et al., 2002).

\section{Recombinant Protein Expression AN4}

Gibson assembly was used to assemble the AN4-HIS sequence with the pET-21a(+) vector (Novagen) (Gibson et al., 2009). The AN4 open reading frame followed by a Gly3-linker and a His6-tag was used as a template for PCR. Forward and reverse primers were used to add Gibson assembly sites 
(Supplementary Table 5). The vector backbone of the pET21a vector was amplified in a PCR reaction with the following components: $5 \times$ Q5 reaction buffer, $2 \mathrm{mM}$ dNTP mix, 0.1-1 ng pET21a, Q5 high fidelity DNA polymerase, water, forward and reverse primer $(10 \mu \mathrm{M})$ (Supplementary Table 5). Linearization of the vector backbone was performed by $D p n \mathrm{I}$ restriction $\left(1 \mathrm{~h}\right.$ at $\left.37^{\circ} \mathrm{C}\right)$. PCR products were purified with the InnuPREP PCR pure kit (Analytik Jena, Germany). For the Gibson assembly reaction, equimolar amounts of linear vector backbone and AN4 expression construct were mixed together with $15 \mu \mathrm{l}$ of Gibson master mix and incubated at $50^{\circ} \mathrm{C}$ for $1 \mathrm{~h}$.

Half of the Gibson assembly mixture was transformed into heat shock competent E. coli strain Rosetta(DE3) cells (Novagen). Transformants were grown on LB agar plates supplemented with $100 \mu \mathrm{g} / \mathrm{mL}$ ampicillin and $25 \mu \mathrm{g} / \mathrm{mL}$ chloramphenicol, and screened by colony PCR using primers that contain the Gibson assembly sites (Supplementary Table 5). The pET21a plasmids containing the AN4-HIS were purified using the GeneJET Plasmid Miniprep kit (Life Technologies) and sequenced by LGC Genomics (Berlin, Germany) with a forward sequencing primer on pET21a (Supplementary Table 5).

Recombinant $E$. coli Rosetta(DE3) cells were grown overnight in $5 \mathrm{~mL}$ LB supplemented with $20 \mu \mathrm{g} / \mathrm{mL}$ ampicillin and $25 \mu \mathrm{g} / \mathrm{mL}$ chloramphenicol at $37^{\circ} \mathrm{C}$ on a rotary shaker $(185 \mathrm{rpm})$. The next morning, the E. coli cells were diluted in $50 \mathrm{ml}$ LB supplemented with $200 \mu \mathrm{g} / \mathrm{mL}$ carbenicillin and $25 \mu \mathrm{g} / \mathrm{mL}$ chloramphenicol, and grown at $30^{\circ} \mathrm{C}$ on a rotary shaker $(185 \mathrm{rpm})$. After the cells reached an $\mathrm{OD}_{600 \mathrm{~nm}}$ of $0.5-0.6$, the expression of recombinant protein was induced with $0.2 \mathrm{mM}$ IPTG. After application of IPTG, the E. coli cells were grown overnight at $14^{\circ} \mathrm{C}$ on a rotary shaker $(185 \mathrm{rpm})$. When growing bacterial cultures in larger volumes $(300 \mathrm{~mL})$, the time of induction was prolonged to 3 days to obtain more recombinant protein. Next, the E. coli cell cultures were harvested by centrifugation at $8,000 \mathrm{rpm}$ for $15 \mathrm{~min}$. The cell pellets were kept overnight at $-20^{\circ} \mathrm{C}$ and solubilized in $10 \mathrm{~mL}$ of $1 \times \mathrm{PB}$ (20 mM NaH${ }_{2} \mathrm{PO}_{4} \cdot 2 \mathrm{H}_{2} \mathrm{O}, 230 \mathrm{mM} \mathrm{Na} \mathrm{HPO}_{4}$ ) with $1 \mathrm{M} \mathrm{NaCl}$ and $1 \mathrm{mg} / \mathrm{mL}$ lysozyme at $\mathrm{pH}$ 8.0. Additionally, the cells were sonicated three times for $2.5 \mathrm{~min}$. After sonication, the solutions were centrifuged at $4^{\circ} \mathrm{C}$ for $45 \mathrm{~min}(9,000 \mathrm{rpm})$ to separate the soluble from the insoluble fractions. The insoluble fractions were resuspended in $8 \mathrm{M}$ urea. Both fractions were checked for the presence of recombinant protein by SDS - PAGE and Western blot analysis.

Escherichia coli cell extracts containing the recombinant AN4-HIS were used for further purification. Therefore extracts adjusted to $10 \mathrm{mM} \mathrm{IZ}$ and $\mathrm{pH} 8.0$, were loaded on a Ni-NTA agarose column (MCLAB, South San Francisco, CA, United States) equilibrated with $\mathrm{PB}$ pH 8.0 containing $1 \mathrm{M} \mathrm{NaCl}$. The column was washed with $\mathrm{PB}$ containing $1 \mathrm{M} \mathrm{NaCl}, 50 \mathrm{mM}$ IZ, pH 8.0 and $\mathrm{PB}$ containing $1 \mathrm{M} \mathrm{NaCl}, 75 \mathrm{mM}$ IZ, pH 8.0 to remove a specifically bound proteins. Elution of the column was performed with $\mathrm{PB}$ containing $1 \mathrm{M} \mathrm{NaCl}, \mathrm{pH} 8.0$ with increasing concentrations of IZ ranging from 100 to $500 \mathrm{mM}$ IZ. Several fractions of $500 \mu \mathrm{L}$ were collected for each elution buffer. The $\mathrm{OD}_{280 \mathrm{~nm}}$ of each fraction was measured with the Nanodrop 2000 spectrophotometer (Thermo Scientific). The purity of the protein samples was verified by SDS-PAGE and Western blot analysis.

Protein samples were analyzed by SDS-PAGE on $15 \%$ acrylamide gels as described by Laemmli (1970). After separation, proteins were visualized by gel staining with Coomassie Brilliant Blue R-250 or blotted onto polyvinylidene fluoride transfer membranes (FluoroTrans ${ }^{\circledR}$ PVDF, Pall Laboratory, United States). Membranes were blocked with TBS (10 mM Tris, $150 \mathrm{mM} \mathrm{NaCl}, 0.1 \%$ (v/v) Triton X-100, pH 7.6) containing $5 \%(\mathrm{w} / \mathrm{v})$ non-fat milk powder. Subsequently, membranes were incubated for $1 \mathrm{~h}$ with a mouse monoclonal anti-His6 antibody (Thermo Fisher Scientific) diluted 1:3000 in TBS. After washing three times with TBS, membranes were incubated for $1 \mathrm{~h}$ with the 1:1000 diluted rabbit anti-mouse IgG secondary antibody labeled with horseradish peroxidase (Dako, Glostrup, Denmark). After washing twice with TBS and once with $0.1 \mathrm{M}$ Tris- $\mathrm{HCl}$ buffer ( $\mathrm{pH}$ 7.6), immunodetection was achieved using a colorimetric assay with $0.1 \mathrm{M}$ Tris- $\mathrm{HCl}$ buffer $(\mathrm{pH} 7.6)$ containing $700 \mu \mathrm{M}$ 3,3'-diaminobenzidine tetrahydrochloride (Sigma-Aldrich, St. Louis, MO, United States) and $0.03 \%(\mathrm{v} / \mathrm{v})$ hydrogen peroxide. The detection reaction was stopped after $2-10 \mathrm{~min}$ by washing the membrane with distilled water. All washes and incubations were performed at room temperature on a gently shaking platform.

\section{Pull-down Analysis}

Pull-down assays were performed on column. First, the soluble fractions of the two different $E$. coli cultures harboring the AN4-HIS pET expression vector (treated or non-treated with IPTG) were incubated with the $\mathrm{Ni}$ matrix overnight. After washing, the Ni-agarose beads were incubated with leaf extracts from 16-day-old Arabidopsis plants subjected or not subjected to $\mathrm{NaCl}$ stress for $5 \mathrm{~h}$, as described in Section "Hormone and Abiotic Stress Treatments," resulting in four different combinations. For each pull-down experiment three replicates were performed and analyzed by mass spectrometry.

First, the soluble fraction $(50 \mathrm{~mL})$ of the E. coli culture producing recombinant AN4-HIS was incubated overnight with the equilibrated $\mathrm{Ni}$ matrix $(100 \mu \mathrm{L})$. The soluble fraction originated from a $300 \mathrm{~mL}$ E. coli culture induced or non-induced with IPTG to produce the recombinant AN4 (bait). After overnight incubation, the mixture of $\mathrm{Ni}$-agarose beads and soluble E. coli protein solution was transferred in a Poly-Prep purification column (Bio-Rad). The column was washed first with $\mathrm{PB}$ containing $1 \mathrm{M} \mathrm{NaCl}$ and $50 \mathrm{mM}$ IZ at $\mathrm{pH} 8.0$ followed by a second wash with the same buffer, but with $75 \mathrm{mM}$ IZ. Then the $\mathrm{Ni}$-agarose beads were transferred to an Eppendorf tube, $1 \mathrm{~mL}$ plant extract (prey) was added to the beads and this mixture was incubated for 30-35 min on a turning wheel. The plant extract was obtained from untreated or salt-stressed Arabidopsis plant material $(0.5 \mathrm{~mL})$ with $1 \mathrm{~mL}$ extraction buffer $(1 \times \mathrm{PB}, 1 \mathrm{M} \mathrm{NaCl}$, $25 \mathrm{mM}$ IZ, 10\% glycerol, 0.1\% Tween-20, 1\% $\beta$-mercaptoethanol and complete ${ }^{\mathrm{TM}}$, Mini, EDTA-free Protease Inhibitor Cocktail at $\mathrm{pH}$ 8.0). After centrifugation and removal of the plant extract, the beads were washed with the first wash buffer containing $50 \mathrm{mM}$ IZ. Finally, the beads were washed three times with $20 \mathrm{mM}$ Tris- $\mathrm{HCl}$ containing $2 \mathrm{mM} \mathrm{CaCl}_{2}$ at $\mathrm{pH} 8.0$ and resuspended in $150 \mu \mathrm{L}$ of the same buffer to store at $-20^{\circ} \mathrm{C}$ prior to mass 
spectrometry. All steps described above were performed in a cold room at $4^{\circ} \mathrm{C}$.

Before mass spectrometry, the samples were treated with trypsin $(1 \mu \mathrm{g})$ for $4 \mathrm{~h}$ at $37^{\circ} \mathrm{C}$ to cleave all proteins from the Ni beads. After removal of the Ni beads, a second trypsin treatment was performed overnight at $37^{\circ} \mathrm{C}$. Trifluoroacetic acid (1\%) was added to deactivate the trypsin and the samples were subsequently desalted. Next, the samples were dried completely and re-dissolved in $2 \%$ acetonitrile and $0.1 \%$ trifluoroacetic acid. Afterward, mass spectrometry was performed with the Q Exactive ${ }^{\mathrm{TM}}$ HF Hybrid QuadrupoleOrbitrap ${ }^{\mathrm{TM}}$ Mass Spectrometer. Database searches were achieved with the MaxQuant software and statistical analysis with the Perseus software. Both analyses were outsourced to the Proteomics Expertise Center (Center for Medical Biotechnology, VIB, UGhent).

\section{Confocal Microscopy and Image Analysis}

Microscopy analysis was performed using the confocal laser scanning microscope Nikon A1R (Nikon Belux). The lower epidermis of the leaf disks (spots that are infiltrated) were visualized using the $40 \times \mathrm{S}$ Plan Fluor ELWD objective lens (NA 0.6). All images are a combination of different fluorescent images acquired along the $z$-axis, as such the complete epidermis cell could be visualized. EGFP was excited with a $488 \mathrm{~nm}$ argon ion laser, fluorescent emission filters were $525 \mathrm{~nm}$ for EGFP and $650 \mathrm{~nm}$ for autofluorescence of chlorophyll. All images were created by the software package NIS-Elements (Nikon) and image analysis was performed using Fiji (Schindelin et al., 2012).

\section{In Silico Tools}

Multiple sequence alignments were performed with Clustal Omega $^{2}$ and pairwise sequence alignments were obtained using EMBOSS Water ${ }^{3}$. Prediction of the presence of a signal peptide was conducted using Phobius and SignalP 4.1 (Käll et al., 2004; Petersen et al., 2011), whereas the presence of a NLS was predicted by NucPred (Brameier et al., 2007). Using SUBA3, protein subcellular localization was predicted (Hooper et al., 2014). BLASTn and tBLASTn searches against the expressed sequence tag (EST) database were performed using the NCBI website ${ }^{4}$. Using the eFP browser (Winter et al., 2007) and Genevestigator (Hruz et al., 2008), microarray expression data were analyzed to study the developmental expression as well as the gene expression under different stress conditions.

\section{RESULTS}

\section{Nictaba Homologs in A. thaliana}

This study focuses on three Nictaba homologs from Arabidopsis, namely AN3 (AT4G19850.2), AN4 (AT1G31200), and AN5 (AT4G19840). These ArathNictaba sequences contain next to

\footnotetext{
${ }^{2} \mathrm{http}: / /$ www.ebi.ac.uk/Tools/msa/clustalo/

${ }^{3}$ http://www.ebi.ac.uk/Tools/psa/emboss_water/

${ }^{4}$ https://blast.ncbi.nlm.nih.gov
}

their Nictaba domain (approximately $145 \mathrm{AA}$ ), an N-terminal sequence of different length (Figure 1A). Protein BLAST with these $\mathrm{N}$-terminal sequences concedes no homology to any known protein domain. A search for signal peptides and transmembrane regions using the Phobius and SignalP 4.1 server predicted no signal peptides or transmembrane regions. Judging from the absence of signal peptides, AN3, AN4, and AN5 are probably synthesized on free ribosomes in the cytoplasm.

Assuming that the primary transcripts undergo no further processing, AN3, AN4, and AN5 will differ from each other in their molecular masses and isoelectric points. AN5 is the largest protein with a molecular mass of $28.1 \mathrm{kDa}$. AN3 has a molecular mass of $25.6 \mathrm{kDa}$ and AN4 is the smallest protein, being $20.3 \mathrm{kDa}$. AN3 is an acidic protein with an isolectric point of 6.11 whereas both AN4 and AN5 have an isoelectric point around 9 (9.44 and 9.18, respectively).

Sequence alignment of the Nictaba domains for the ArathNictaba 3-5 and Nictaba from tobacco (encoded by AF389848) allowed to calculate the sequence identities and similarities (Figure 1B). AN3 and AN5 show the highest percentage of sequence identity ( $48.4 \%$ sequence identity; $67.5 \%$ sequence similarity) in the lectin domain sequence. Moreover, their sequence identity with Nictaba is very similar, 31.8 and $31.5 \%$, respectively. The lowest percentage of sequence identity with Nictaba is observed for AN4 (22.9\%). The tryptophan residues reported to be important for carbohydrate binding activity of Nictaba are conserved in all ArathNictaba sequences (Figure 1B, Schouppe et al., 2010).

\section{ArathNictabas Locate to the (Nucleo)Cytoplasmic Compartment}

To validate the in silico results with respect to the cellular localization, $\mathrm{N}$ - and C-terminal EGFP fusion constructs were created. Microscopy analysis of the T2 generation of Arabidopsis plants stably transformed with the C-terminal EGFP fusion constructs for each ArathNictaba sequence showed a nucleocytoplasmic localization for AN4 and AN5 whereas AN3 only resided in the cytoplasm (Figure 2). The localization of AN4 and AN5 in the cytoplasm and the nucleus was confirmed for the N-terminal EGFP fusion constructs. The N-terminal EFGP fusion construct of AN3 never showed a fluorescent signal (Supplementary Figure 1A).

In addition, leaves from $N$. benthamiana were transiently transformed with the $\mathrm{N}$ - and C-terminal EGFP fusion constructs for each ArathNictaba sequence and confirmed the results obtained with A. thaliana (Supplementary Figure 1B). In both experiments, free EGFP was used as a positive control and was observed in the nucleus and the cytoplasm (Supplementary Figures 1A,B).

\section{Expression of the ArathNictaba Genes during Development of WT A. thaliana Plants}

Using RT-qPCR, the expression level of the ArathNictabas was investigated in different tissues from Arabidopsis during development under standard growth conditions. Plant material 


\section{A}

AN3

Nictaba

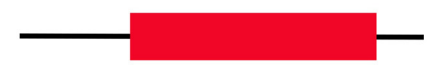

Nictaba

AN4

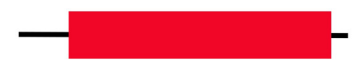

180 aa

220 aa

\section{AN5}

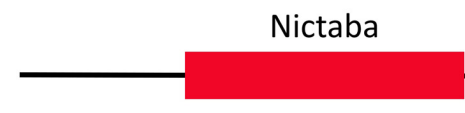

246 aа

100 aа
B

MQGQWIAARDLSITWVDN--PQYWTWKTV------DPNIEVAELRRVAWLDIYGKIETKN KNCEMLYARDLS ITWAESQTNKYWSWFSDLDQTSSDVRTEVAKMERVAWLEVVGKFETEK --------SGLNFVWGGD--SRYWVI PKEP--------RMPAELKMVSWLEVTGSFD--K SNCFMLFAKNLS ITWSDD--VNYWTWFTEKESPNEN--VEAVGLKNVCWLDITGKFDTRN

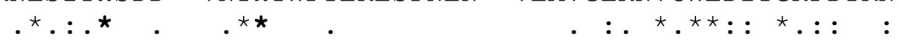

Nictaba AN3

AN4 AN5

Nictaba

AN3

AN4

AN5

Nictaba

AN3

AN4

AN5

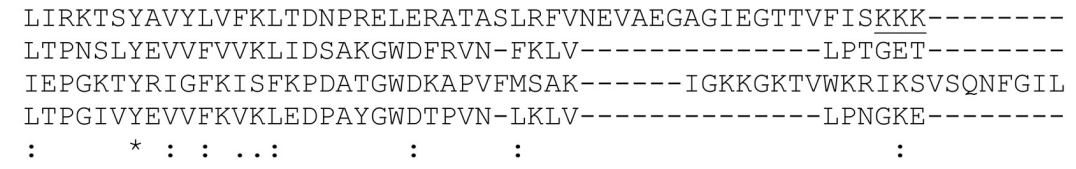

KLPGELGRFPHLRSDGWLEIKLGEFFNNLGE-DGEVEMRLMEINDKTWKSGIIVKGFDIR $\bar{K}$ ERRENV--NLLERNKWVEI PAGEFMISPEHLSGK̄ IEIRK-----------------KGGSEPVNIP-DESDGLFEILVSP---TALNQDTKLQFGLYEVVWTGRWKTGLLIH----KPQEKKVSLRELPRYKWVDVRVGEFVPEKSA-AGEITFSMY $\underline{\text { E} H A A G V W K K G L S L K G V A I R ~}$ * : . : : . $\bar{\vdots}:$ :

PN
--
--
PK

FIGURE 1 | Domain architectures of AN3, AN4, and AN5 (A), and protein sequence alignment of the Nictaba sequence and the Nictaba domains of the ArathNictabas (B). (A) The domain architectures are drawn to scale. The scale bar represents 100 AA. (B) The tryptophan residues important for carbohydrate binding in Nictaba are marked in bold (Trp15, Trp22). The glutamic acid residues (Glu138 and Glu145) are marked in bold and underlined. The NLS of Nictaba is underlined.

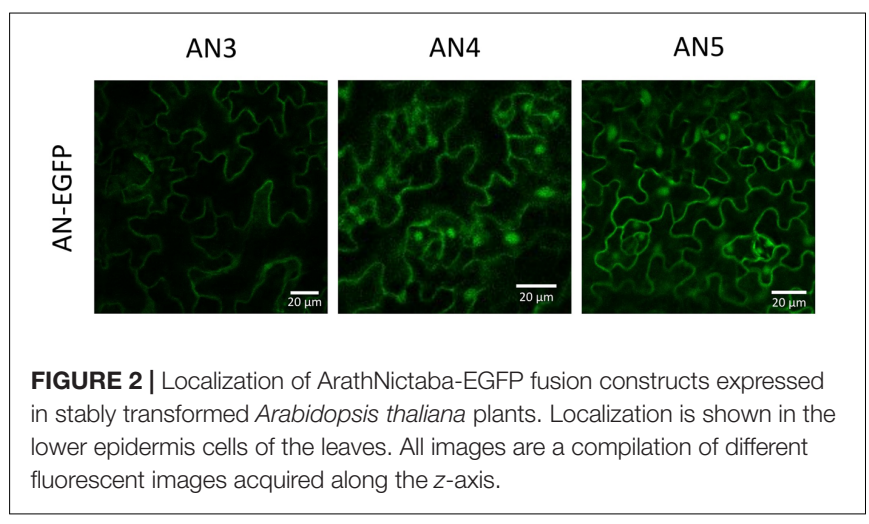

was collected at different developmental stages starting from 6-day-old plantlets to 54-day-old plants and the normalized relative expression for the three ArathNictabas was quantified throughout the development of the plant relative to the expression of these ArathNictabas in 6-day-old plantlets (Figure 3). Transcripts for the three ArathNictaba genes are detected in every tissue at all developmental stages tested. The expression level of $A N 3$ is significantly higher in the stems and the flowers compared to the expression in 6-day-old plantlets. The expression level of AN4 is slightly but significantly higher in the roots and significantly lower in the flowers of the plant compared to its expression in 6-day-old plantlets. The expression level of AN5 is significantly higher in the rosette- and cauline leaves at all developmental stages tested. Moreover, the expression level of AN5 is slightly but significantly lower in the flowers compared to its expression in 6-day-old plantlets.

The normalized expression of AN3, AN4, and AN5 compared to the expression of one of the three reference genes ( $P P 2 A$, TIP41, or UBC9) is much lower for AN3 and AN4 than for AN5, indicating that the expression level of AN5 is higher than the expression levels of $A N 3$ and AN4 in all tissues throughout the development of the plant (data not shown). 


\section{ArathNictaba Expression Is Stress-Inducible \\ AN3, AN4, and AN5 Are Differentially Expressed in Response to Hormone Treatments}

Sixteen-day-old Arabidopsis seedlings were subjected to different hormone treatments in particular $100 \mu \mathrm{M}$ MeJA, $100 \mu \mathrm{M} \mathrm{ABA}$, and $300 \mu \mathrm{M}$ SA. Transcript levels for AN3, AN4 and AN5 were determined by qPCR analysis (Figure 4). Control genes known to be responsive to MeJA (JMT), ABA (Cor15A), and SA (WRKY70) treatments are significantly upregulated indicating that the plants sensed the different stress treatments.

The expression of $A N 3$ is significantly upregulated after treatment with different hormones with a fourfold upregulation after 5, 10, and $24 \mathrm{~h}$ of MeJA treatment. After ABA and SA treatment, the upregulation of the expression of $A N 3$ is less pronounced. ABA treatment resulted in a twofold upregulation after 5 and $10 \mathrm{~h}$, SA treatment yielded a 1.6-3.6-fold upregulation after $1,3,5,10$, and $24 \mathrm{~h}$.

In contrast with the expression of $A N 3$, the expression of AN4 is mostly downregulated after treatment with different hormones with a 2-2.5-fold downregulation after 5, 10, and $24 \mathrm{~h}$ of MeJA treatment. Whereas a twofold upregulation of the expression of AN3 was observed after 5 and $10 \mathrm{~h}$ of ABA treatment, the expression of $A N 4$ is approximately two times significantly downregulated. After SA treatment, the expression of AN4 is not changed except for a small significant downregulation after $10 \mathrm{~h}$.

The expression of AN5 is only weakly influenced by the MeJA treatment. Similar to the expression of $A N 3$, there is a twofold significant upregulation of the expression of AN5 after 5 and $10 \mathrm{~h}$ of ABA treatment. After SA treatment, the expression of AN5 is not changed except for a small significant upregulation after $10 \mathrm{~h}$.

\section{The Expression of the ArathNictabas Showed Dissimilar Patterns after Abiotic Stress Treatments}

Transcript levels for AN3, AN4, and AN5 were determined in 16-day-old Arabidopsis seedlings subjected to salinity (150 mM $\mathrm{NaCl}$ ) and heat stress (Figure 5). Control genes known to be responsive to salt $(R D 29 A)$ and heat $(H s p 70 b)$ stress are significantly upregulated indicating that the plants sensed the different abiotic stress treatments. The expression level of AN3 is not affected by salt stress but is 4-6 times significantly upregulated by heat stress after $3,5,10$, and $24 \mathrm{~h}$. Overall, the expression of AN4 is two times downregulated after salt as well as heat stress. The expression of AN5 is only slightly influenced by salt stress and showed a threefold significant downregulation after 10 and $24 \mathrm{~h}$ of heat stress.

\section{Expression of the ArathNictabas after Different Biotic Stresses}

Five-week-old Arabidopsis plants were subjected to infection assays with $P$. syringae or $B$. cinerea, and $M$. persicae infestation. Mock/biotic stress treated samples were collected 0-7 days post-infection/infestation (dpi) and transcript levels for AN3, AN4, and AN5 were determined (Figure 6). qPCR analysis revealed an early 2-2.5-fold upregulation (1-3 dpi) of the expression of AN3 after $P$. syringae infection and a late twofold
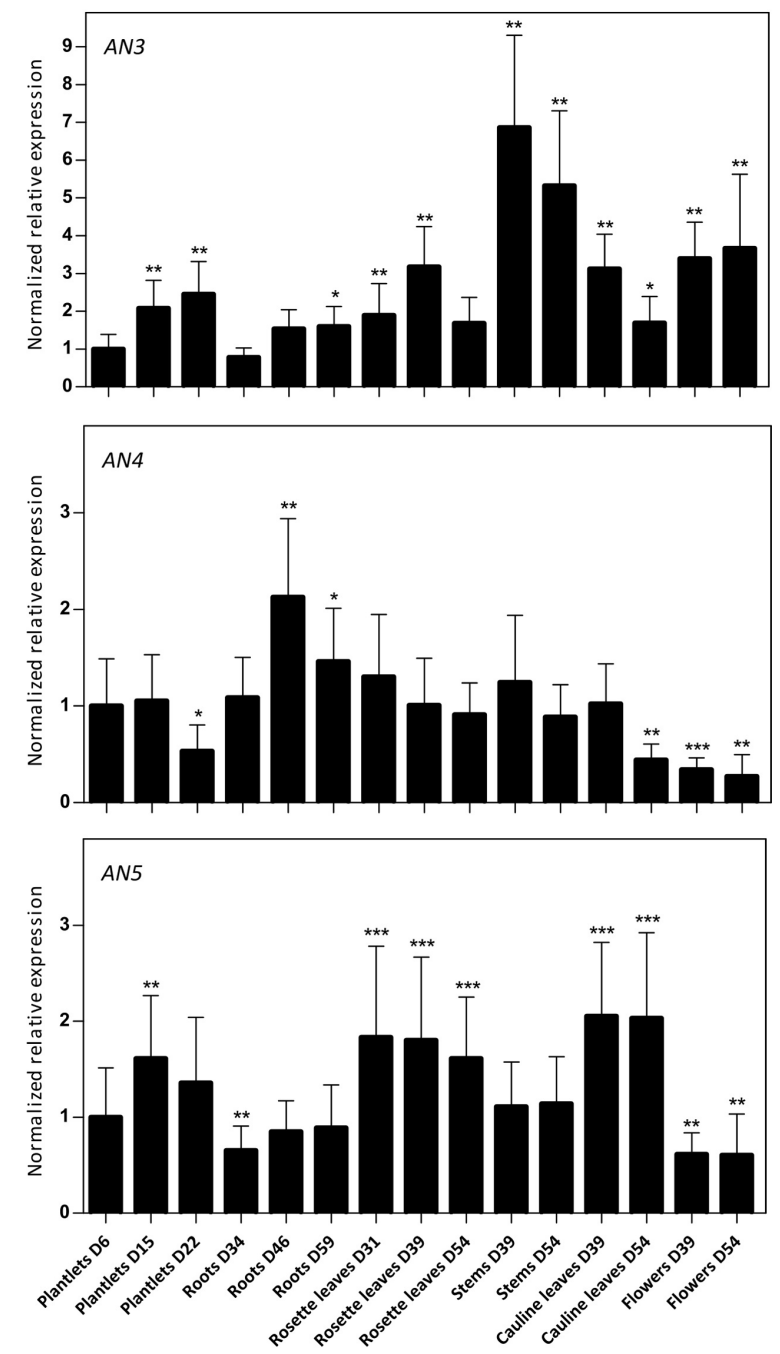

FIGURE 3 | Normalized relative expression of ArathNictaba genes during the development of $A$. thaliana. The normalized transcript levels are the result of two independent biological replicates $(N=2)$. They are presented relatively to the ArathNictaba expression level determined in 6-day-old plantlets. Bars represent the mean $\pm \mathrm{SE}$ normalized relative expression and asterisks indicate statistically significant differences to the expression level of ArathNictaba in 6 -day-old plantlets $\left({ }^{*} p \leq 0.05,{ }^{* *} p \leq 0.01,{ }^{* * *} p \leq 0.001\right.$; REST analysis).

upregulation (5 -7 dpi) of the expression of AN4. However, the expression of AN5 did not change after P. syringae infection. Fungal infection with $B$. cinerea affected ArathNictaba expression levels only weakly (Supplementary Figure 2). A small but significant downregulation of the transcript levels for AN5 was observed at 2 and $3 \mathrm{dpi}$. In contrast infestation with $M$. persicae revealed an almost twofold upregulation of the transcript levels for AN5 after 3 days. The expression of AN4 is not affected by infestation of the plants with $M$. persicae whereas the expression of AN3 shows a twofold downregulation after 3 days. Control genes known to be responsive to $P$. syringae $(P R 1), B$. cinerea (PDF1.2), and $M$. persicae (PR1) are significantly upregulated, indicating that the plants sensed the different biotic stress treatments. 


\section{$100 \mu \mathrm{M}$ MeJA}
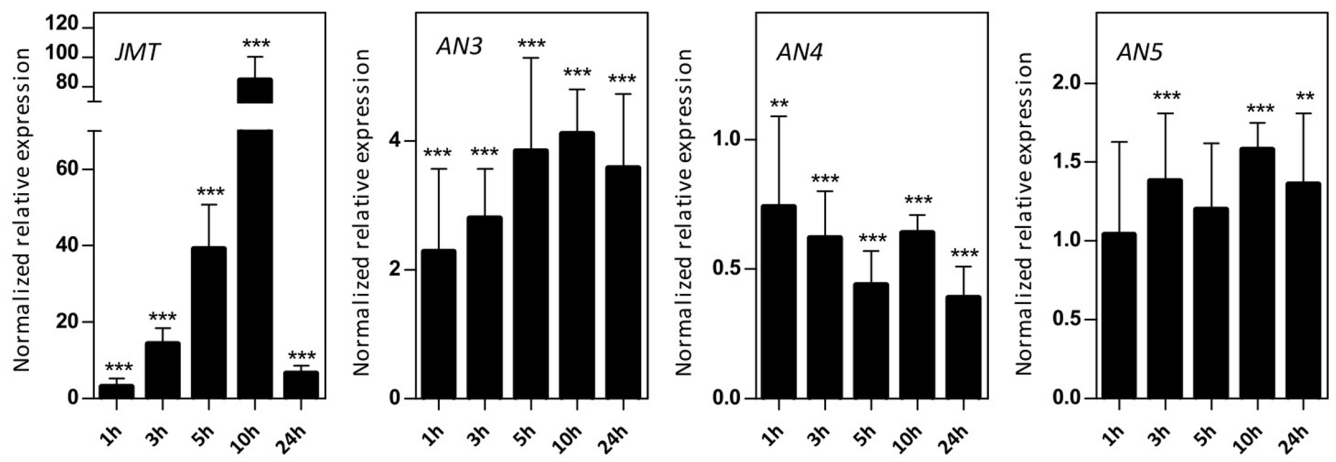

$100 \mu \mathrm{M}$ ABA
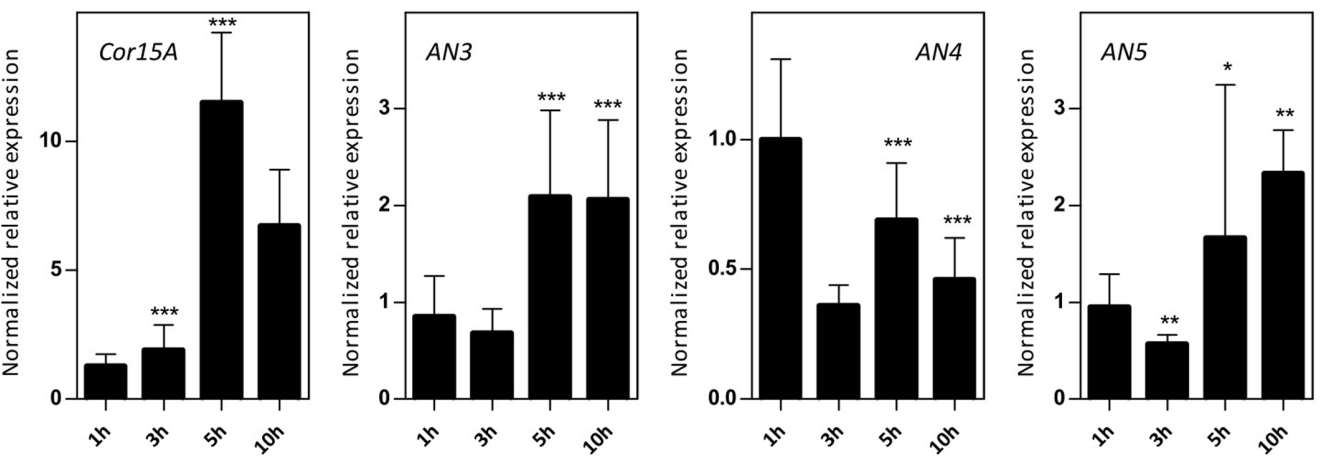

\section{$300 \mu \mathrm{M}$ SA}
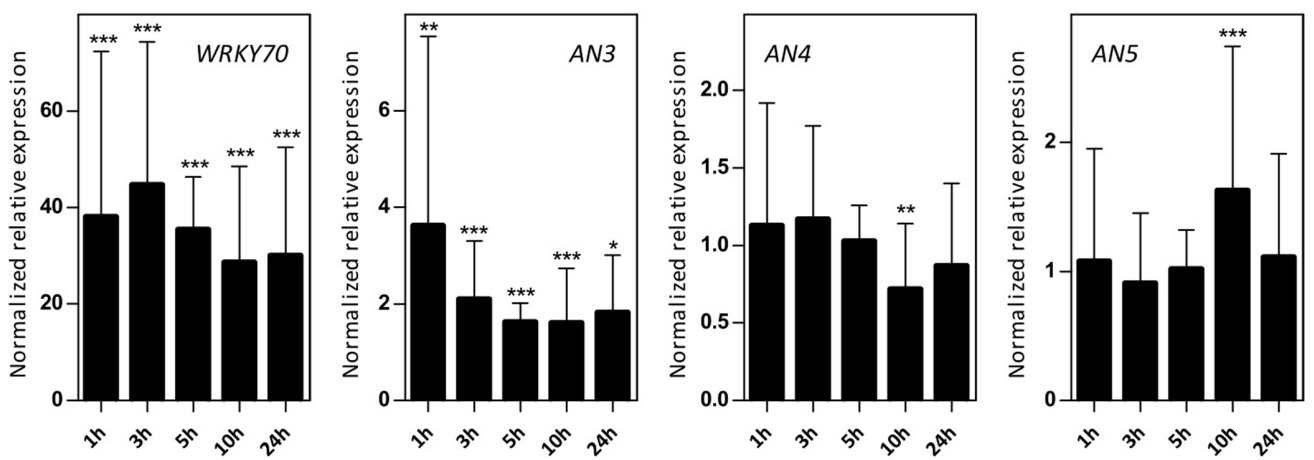

FIGURE 4 | Normalized relative expression of ArathNictaba genes after MeJA, ABA, and SA hormone treatment. The normalized transcript levels are the result of two or four independent biological replicates ( $N=2$ for $A B A, N=4$ for MeJA and SA). They are presented relatively to the ArathNictaba expression level determined in the mock treated plantlets. Bars represent the mean \pm SE normalized relative expression and asterisks indicate statistically significant differences to the expression level of ArathNictaba in mock treated plantlets $\left({ }^{*} p \leq 0.05,{ }^{* *} p \leq 0.01,{ }^{* * *} p \leq 0.001\right.$; REST analysis). The normalized relative expression levels for the control genes for each stress are presented in the left panels.

\section{ArathNictaba Overexpression Lines Show Less Disease Symptoms and Bacterial Growth after $P$. syringae Infection}

To further investigate the biological importance of the ArathNictabas in the stress response, $A$. thaliana was transformed with the overexpression constructs 35S::AN4 and 35S::AN5, and transgenic lines were selected. Transcript levels for AN4 and AN5 were determined in the homozygous AN4 and AN5 overexpression lines using RT-qPCR on cDNA obtained from 15-day-old Arabidopsis seedlings. The 35S::AN4 transgenic lines show 50- to 800-fold expression of AN4 compared to WT plants. However, for the 35S::AN5 transgenic lines a much lower 


\section{$150 \mathrm{mM} \mathrm{NaCl}$}
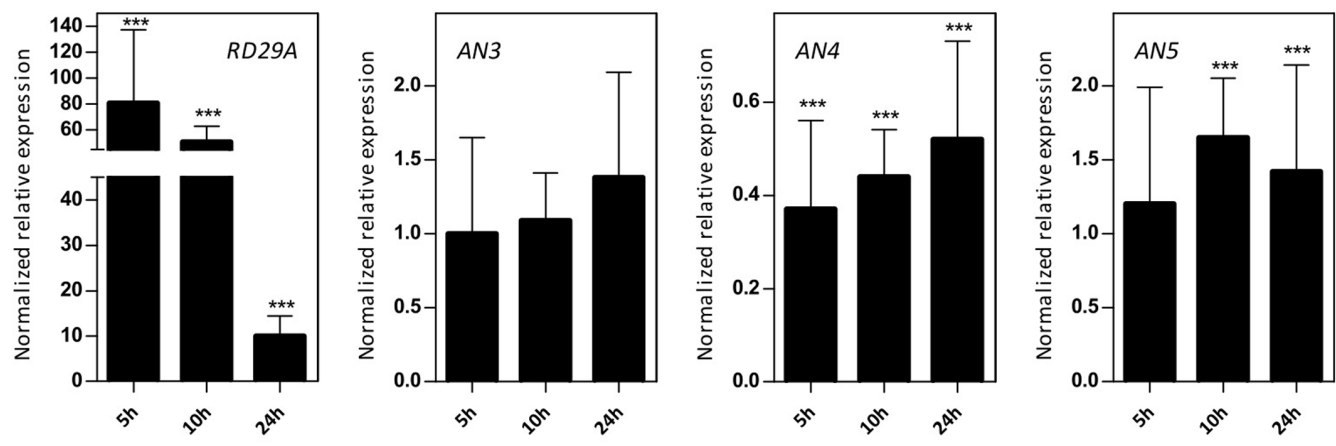

\section{Heat $\left(37^{\circ} \mathrm{C}\right)$}
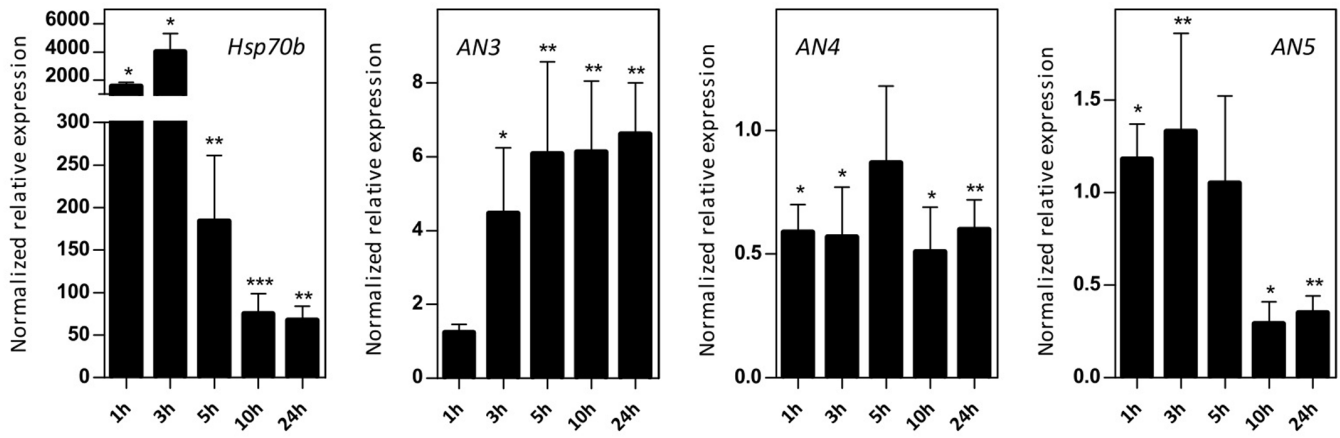

FIGURE 5 | Normalized relative expression for ArathNictaba genes after salt and heat stress. The normalized transcript levels are the result of two or four independent biological replicates ( $N=2$ for heat stress, $N=4$ for salt stress). They are presented relatively to the ArathNictaba expression level determined in the mock treated plantlets. Bars represent the mean \pm SE normalized relative expression and asterisks indicate statistically significant differences to the expression level of ArathNictaba in mock treated plantlets $\left({ }^{*} p \leq 0.05,{ }^{* *} p \leq 0.01,{ }^{* * *} p \leq 0.001\right.$; REST analysis). The normalized relative expression levels of the control genes for each stress are presented in the left panels.

overexpression level is observed, ranging between 15 - and 45 -fold compared to WT plants. Based on their expression level relative to the ArathNictaba expression in WT plants, three independent overexpression lines exhibiting different overexpression levels were selected (Figure 7).

Wild type and transgenic 35S::AN4 and 35S::AN5 A. thaliana plants were infected with the virulent hemibiotrophic $P$. syringae to investigate the role of the ArathNictaba genes in the defense against this pathogen. Infection of $A$. thaliana plants with $P$. syringae results in yellow lesion areas on the rosette leaves of the plant. Leaf damage was measured daily on scanned leaves and the percentage of the lesion area relative to the total leaf area was calculated. First bacterial lesions started to appear at $3 \mathrm{dpi}$, but only at $4 \mathrm{dpi}$ differences in leaf damage were observed for the overexpression lines compared to WT plants (Figure 8A). Statistically significant differences in leaf damage compared to the leaf damage in WT plants were observed especially for lines AN4_B1, AN4_L4, AN5_D5, and AN5_G3. These four overexpression lines reveal a significantly lower percentage of leaf damage compared to WT plants suggesting they are more tolerant to $P$. syringae infection. The leaf damage in mock treated plants was also determined but was never higher than 6.5\% (data not shown). Furthermore, the leaf damage observed for the 35S::AN5 lines is significantly $(p \leq 0.05)$ negatively correlated with the expression level of the different overexpression lines (Pearson correlation, SPSS23). No such correlation between the level of leaf damage and the expression level of AN4 was observed for the different overexpression lines for AN4.

To strengthen these results, $P$. syringae biomass on the A. thaliana WT and transgenic plants was quantified in leaves at 3 and $4 \mathrm{dpi}$. Transgenic lines AN4_B1, AN5_D5, and AN5_G3, which showed a significantly lower percentage of leaf damage compared to WT plants, also had a significantly lower $P$. syringae biomass (Figure $\mathbf{8 B}$ ) indicating that these overexpression lines are more tolerant than WT plants to infection with $P$. syringae. Transgenic line AN5_A2, which had less (though not significantly) leaf damage compared to WT, also revealed a significantly lower $P$. syringae biomass. Transgenic line AN4_L4 had a lower (but not significant) P. syringae biomass compared to WT plants.

A third analysis included the visualization and quantification of cell death in the leaves using trypan blue staining 


\section{P. syringae}
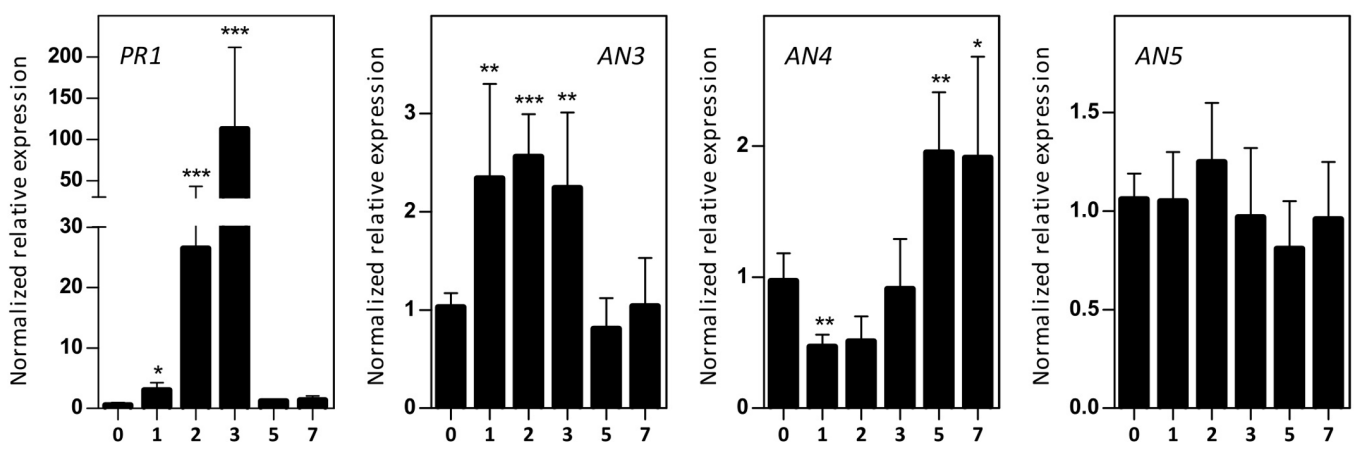

\section{M. persicae}
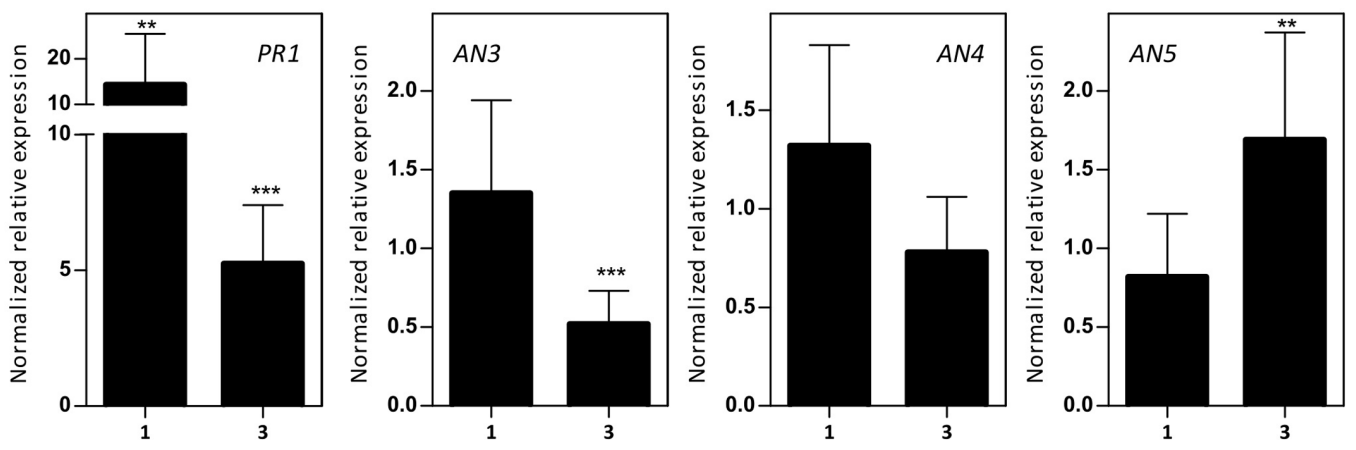

FIGURE 6 | Normalized relative expression for ArathNictaba genes after Pseudomonas syringae infection and $M$. persicae infestation. The normalized transcript levels are the result of three or four independent biological replicates ( $N=3$ for $P$. syringae, $N=4$ for $M$. persicae). They are presented relatively to the ArathNictaba expression level determined in the mock treated plants. Bars represent the mean \pm SE normalized relative expression and asterisks indicate statistically significant differences to the expression level of ArathNictaba in mock treated plants ( ${ }^{*} p \leq 0.05,{ }^{* *} p \leq 0.01,{ }^{* * *} p \leq 0.001$; REST analysis). Numbers on the $x$-axis represent the number of days after infection/infestation. The normalized relative expression levels of the control genes for each stress are presented in the left panels.
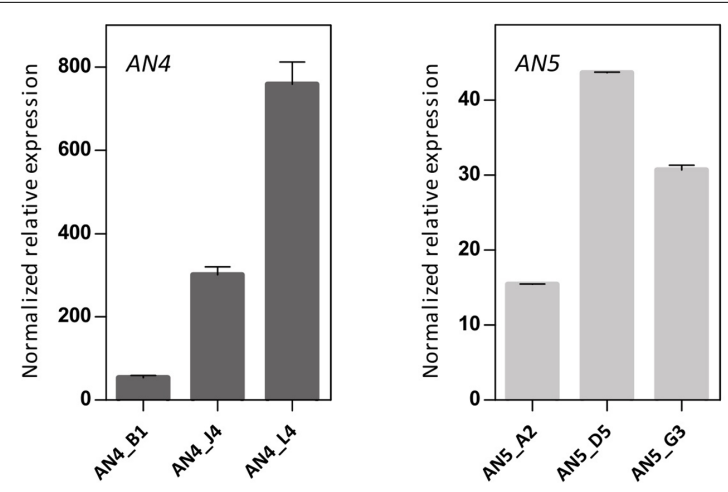

FIGURE 7 | Expression analysis of ArathNictaba overexpression level in 2-week-old 35S::AN4 and 35S::AN5 transgenic lines. Normalized relative expression of AN4 and AN5 compared to WT plants $(N=1)$. Error bars represent standard deviations.

(Figures 8C,D). Transgenic line AN4_B1 showed a significantly lower level of leaf damage and $P$. syringae biomass compared to WT plants (Figures $\mathbf{8 A , B}$ ) which is in agreement with the differences (not significant) observed for cell death (Figure 8C) concluding that line AN4_B1 is more tolerant to $P$. syringae infection than WT plants. Surprisingly, line AN4_J4 showed a significantly reduced amount of cell death compared to WT plants (Figure 8C) but the percentage of leaf damage was almost equal to that of WT plants and the $P$. syringae biomass was significantly higher than in WT plants (Figures 8A,B). Transgenic line AN4_L4 showed comparable results to WT plants in all analyses except for the leaf damage analysis which revealed a significantly lower leaf damage (Figure 8A). As such it cannot be concluded that line AN4_J4 and AN4_L4 are more tolerant to $P$. syringae infection. $P$. syringae biomass for line AN5_A2 is significantly lower than for the WT plants (Figure 8B) which is in agreement with the lower percentages of leaf damage and cell death, suggesting AN5_A2 is more tolerant to $P$. syringae infection than WT plants. Lines AN5_D5 and AN5_G3 show significantly lower levels of leaf damage and $P$. syringae biomass but the amount of cell death is not significantly lower. Therefore lines AN5_D5 and AN5_G3 are probably also more tolerant to $P$. syringae infection than WT plants. The trypan blue staining in mock treated plants 

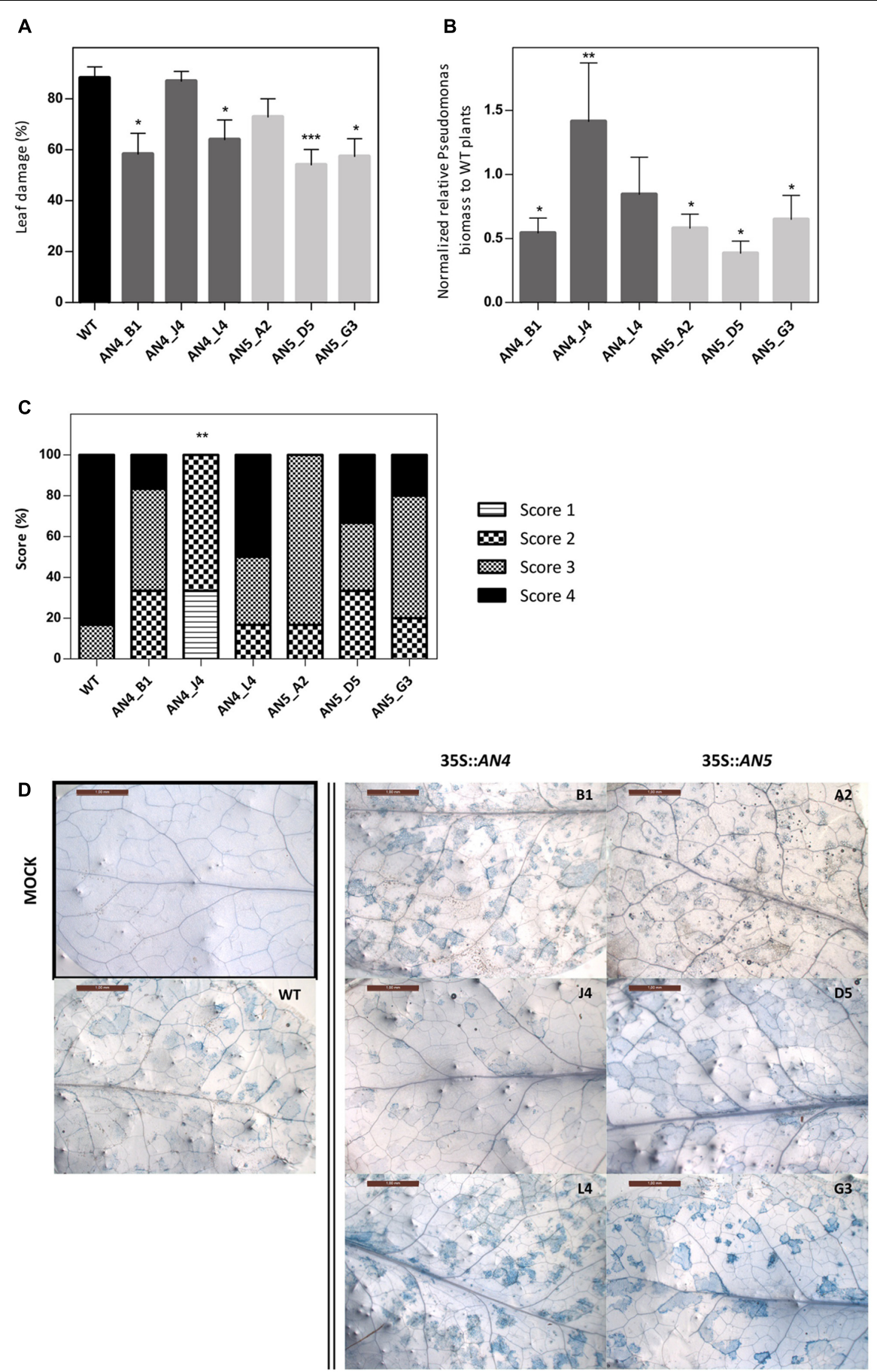

FIGURE 8 | (A) Percentage of leaf damage in WT and transgenic overexpression $A$. thaliana plants infected with $P$. syringae at 4 dpi. (B) Normalized relative P. syringae biomass in the overexpression lines compared to WT plants at 4 dpi. (C) Scoring of trypan blue stained leaves (D) from overexpression lines compared to WT plants at 4 dpi. (A) Percentage of leaf damage calculated with Assess 2.0 at 4 dpi as percent ratio of yellow lesion area relative to the total leaf area. Bars represent the mean $\pm \mathrm{SE}$ of two independent biological replicates with six individual leaves per line per replicate. Asterisks indicate statistically significant differences to the percentage of leaf damage in WT plants $\left({ }^{*} p \leq 0.05,{ }^{* *} p \leq 0.01,{ }^{* * *} p \leq 0.001\right.$; Mann-Whitney $U$ test). (B) Bars represent the mean $\pm \mathrm{SE}$ relative $P$. syringae biomass in overexpression lines to biomass in WT plants at 4 dpi from two independent biological replicates normalized with two $A$. thaliana reference genes (ACT2 and PEX4) in REST-384. Asterisks indicate statistically significant differences to the biomass in WT plants $\left({ }^{*} p \leq 0.05,{ }^{* *} p \leq 0.01,{ }^{* * *} p \leq 0.001\right.$; pair wise fixed reallocation randomization test REST-384). (C) Bars represent the scores in percentage from two independent biological replicates $(N=2)$ with each time three leaves stained per line. Score 1: 0\%, score 2: 1-30\%, score 3: 31-60\% and score 4: 61-100\% trypan blue staining or cell death. Asterisks indicate statistically significant differences to the trypan blue staining in WT plants $\left({ }^{*} p \leq 0.05,{ }^{* *} p \leq 0.01,{ }^{* * *} p \leq 0.001\right.$; Mann-Whitney $U$ test). (D) Representative pictures are shown for each transgenic line. Only one picture of a mock treated leaf is shown (black square). 
was not quantified since no signs of cell death were observed (Figure 8D).

\section{Pull-Down Analysis to Search for Interacting Partners of AN4}

To search for interacting partners, recombinant protein was produced and used for pull-down assays. Recombinant protein production was attempted for AN3, AN4, and AN5 in two eukaryotic systems namely the yeast Pichia pastoris and a tobacco cell culture of bright yellow 2 cells. However, no recombinant protein could be detected for either of the ArathNictabas. Recombinant protein production in E. coli resulted in a small amount of AN4 protein, but no protein for AN3 and AN5 was detected in the soluble fraction. The pull-down experiments were performed with recombinant AN4 produced in E. coli and Arabidopsis extracts from WT seedlings or seedlings treated with $150 \mathrm{mM} \mathrm{NaCl}$ for $5 \mathrm{~h}$. All E. coli and A. thaliana proteins were identified using mass spectrometry. Comparative analyses for pull-down assays performed with the IPTG induced E. coli culture producing AN4 and a non-induced E. coli culture allowed to exclude the E. coli proteins that bound aspecifically to the $\mathrm{Ni}$ matrix and the Arabidopsis proteins that bind aspecifically to these E. coli proteins or to the Ni matrix. Results from the pull-down assays with extracts from non-treated and salt treated plants were also compared with each other. Since the transcript levels for AN4 in Arabidopsis are downregulated after salt stress (see section "The Expression of the ArathNictabas Showed Dissimilar Patterns after Abiotic Stress Treatments") this can also be the case for the interaction partners for AN4. Possible interaction partners for which the expression is downregulated by salt stress are expected to be absent in the analysis using the plant extract from the salt stressed plants compared with the analysis using the extract from the non-treated Arabidopsis seedlings.

Volcano plots allow to quickly identify significant differences between two large datasets by plotting significance ( $p$-value) vs. fold change. A first comparative analysis was made for the pull-down assays performed on $\mathrm{Ni}$ matrix with the IPTG induced and non-induced E. coli culture, using protein extracts of Arabidopsis seedlings grown under normal conditions (Figure 9A). As expected, AN4 is significantly more present in the pull-down experiments performed with the induced E. coli culture. Next to AN4, three A. thaliana proteins originating from the plant extract are also significantly more present in particular TGG1, ESM1, and PTAC16, suggesting these proteins are possible interaction partners for AN4. The volcano plot in Figure 9B shows a second analysis resulting from the comparison between the proteins identified in the pull-down assays performed with the IPTG induced and non-induced E. coli culture, using a plant extract of salt-treated Arabidopsis seedlings. AN4 and two A. thaliana proteins TGG1 and BGLU23 are significantly more present in the pull-down experiments with the induced $E$. coli culture (Figure 9B), suggesting that these two proteins are possible interaction partners for AN4. TGG1 was also retrieved in the first analysis (Figure 9A) making it more conclusive to be a true interaction partner for AN4. Compared to TGG1, the data for BGLU23 had a much lower $p$-value (Figure 9B). A comparison between the datasets resulting from the pull-down assays performed using the IPTG induced E. coli culture and protein extracts from non-treated Arabidopsis seedlings and salt-treated Arabidopsis seedlings did not yield any significant hits, suggesting that there are no significant differences in AN4-bound proteins from plant extracts of the salt stress treated Arabidopsis seedlings compared to WT seedlings (data not shown). All significant hits from the pull-down experiments can be found in Supplementary Tables 6, 7 .

\section{DISCUSSION}

\section{ArathNictabas Are Expressed in the Nucleus and the Cytoplasm}

Alignment of the sequences encoding Nictaba from tobacco and the Nictaba domains from the ArathNictaba sequences showed that the tryptophan residues important for carbohydrate binding activity in Nictaba are conserved in all Nictaba domains. However, based on this observation, no conclusions can be drawn with respect to the carbohydrate binding specificity of the ArathNictabas. Stefanowicz et al. (2012) analyzed the carbohydrate specificity of an F-box Nictaba (AT2G02360) protein from $A$. thaliana. The F-box Nictaba sequence contains the tryptophan residues important for sugar binding activity but glycan array analyses yielded results that were very different compared to the GlcNAc binding Nictaba. F-box Nictaba was shown to specifically bind to $N$-acetyllactosamine, Lewis A, Lewis X, Lewis Y and blood type B motifs (Stefanowicz et al., 2012).

Enhanced green fluorescent protein fusion proteins for AN4 and AN5 localized to the nucleus and the cytoplasm whereas AN3 only resided in the cytoplasm (Figure 10). The nucleocytoplasmic localization for AN5 was previously also reported by Cayla et al. (2015). No other experimental data are available with respect to the subcellular localization of AN3 and AN4, but a cytoplasmic localization is also predicted by the SUBA3 server (Hooper et al., 2014).

The results from the microscopy analyses are in agreement with the absence of a signal peptide in the three ArathNictaba sequences and suggest that translation of the ArathNictaba transcripts takes place on free ribosomes in the cytoplasm. AN4 and AN5 locate to the nucleus although the putative NLS reported for the Nictaba sequence from tobacco (K102-K105) is not conserved in the ArathNictaba sequences. Lange et al. (2007) reported that proteins larger than $40 \mathrm{kDa}$ are too big to diffuse passively into the nuclear compartment, as already shown in Paine et al. (1975). Görlich (1998) reported that the nuclear pore complex allows passive diffusion from proteins up to $60 \mathrm{kDa}$. Wang and Brattain (2007) extended this limit, stating that the size of the proteins that diffuse passively into the nucleus can be even larger than $60 \mathrm{kDa}$. The calculated molecular mass of the EGFP fusion proteins for AN4 and AN5 is 48.5 and $56.3 \mathrm{kDa}$, respectively. Considering the most recent information about the passive diffusion limit, both proteins could enter the nucleus by passive diffusion through nuclear pore complexes 
A

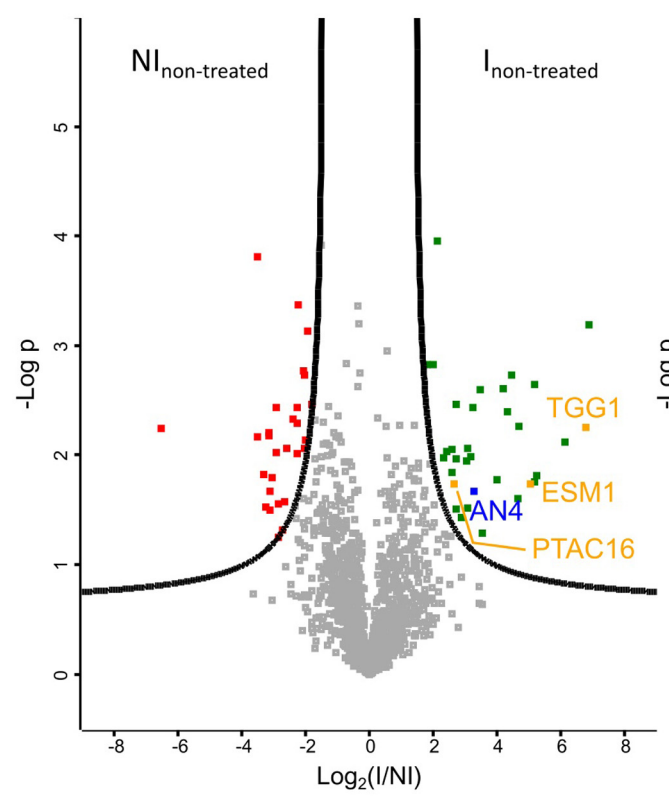

B

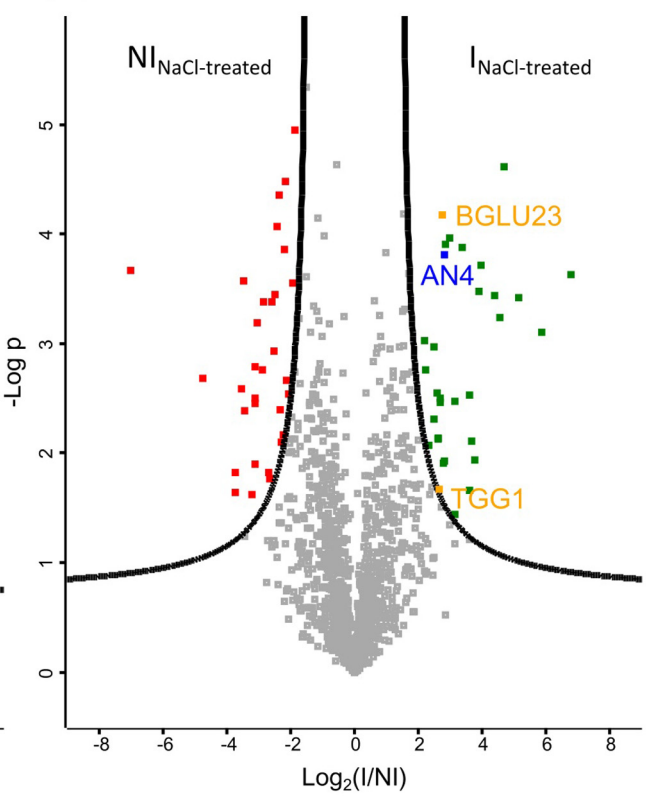

FIGURE 9 | Volcano plots of proteins identified by mass spectrometry $(N=3)$. (A) Plant extract from non-treated Arabidopsis seedlings. FDR = 0.05. (B) Plant extract from Arabidopsis seedlings treated with $150 \mathrm{mM} \mathrm{NaCl}$ for $5 \mathrm{~h}$. FDR $=0.01$. AN4 is indicated in blue, possible interaction partners are shown in orange, Escherichia coli proteins which are significantly more present in the pull-down assays performed with the IPTG treated E. coli culture (I) in green and E. coli proteins which are significantly less present in these pull-down assays are shown in red. NI, non-induced.

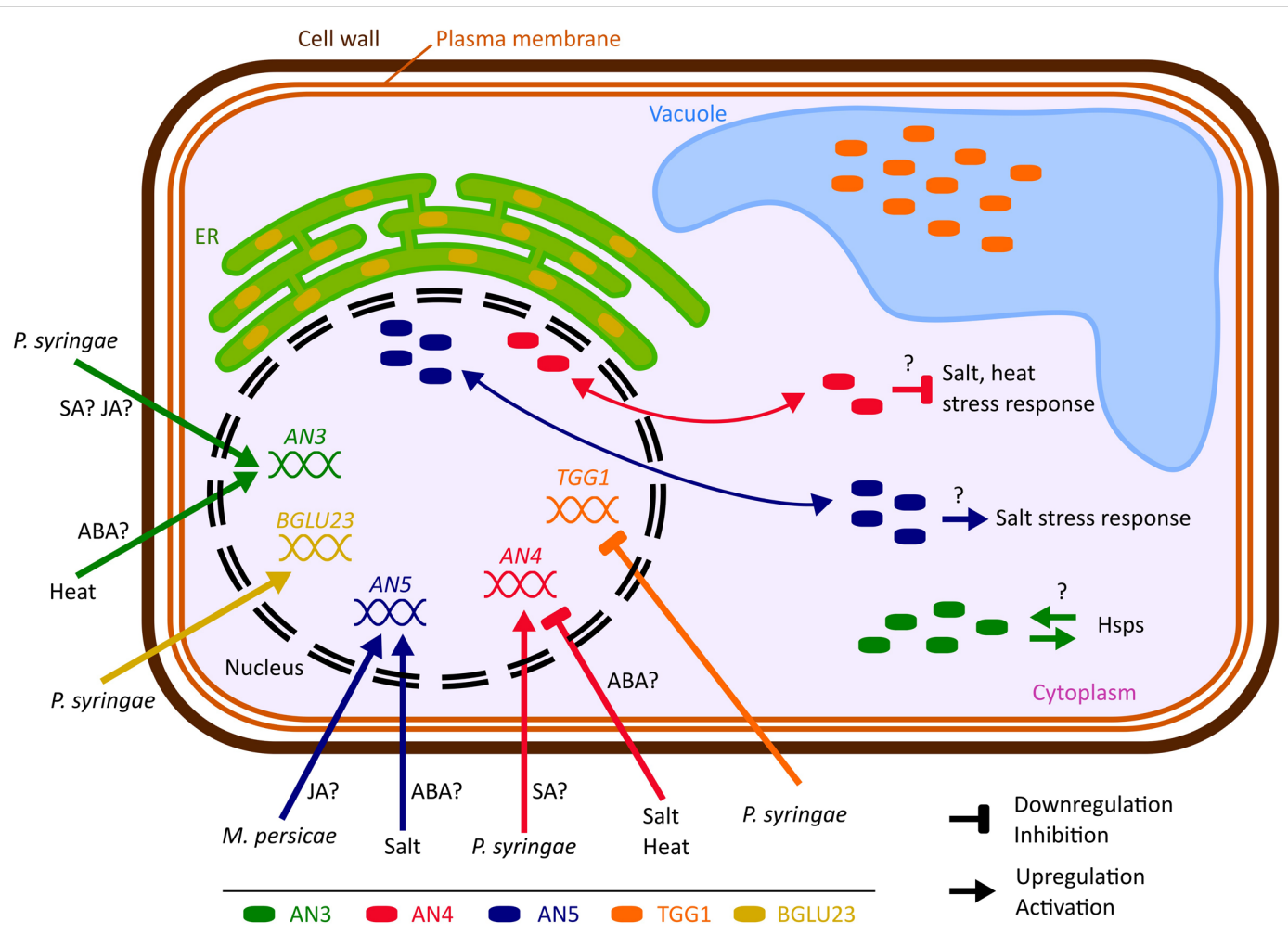

FIGURE 10 | Hypothetical model representing the biological importance of the ArathNictabas in $A$. thaliana cells. The cell and organelles are not drawn to scale. Data available from eFP browser suggest that BGLU23 is upregulated and TGG1 is downregulated after Pseudomonas infection. 
(Wang and Brattain, 2007). Alternatively, AN4 and AN5 possibly contain a non-classical NLS sequence recognized by importin $\alpha$ (Kosugi et al., 2009). Furthermore, additional nuclear import pathways, independent of importin $\alpha$, have been characterized (Suh and Gumbiner, 2003; Ziemienowicz et al., 2003; Pemberton and Paschal, 2005).

Quantitative analyses of the transcript levels for the ArathNictabas in different tissues from A. thaliana revealed that the three ArathNictaba genes under study are expressed in all tissues throughout development. However, it should be mentioned that lectin expression is generally low, especially for AN3 and AN4. However, specific stress factors can enhance the transcript levels for each lectin. The low expression observed for the Arathnictabas is unlike the expression patterns observed for Nictaba from tobacco. The tobacco lectin was not detected in plants grown under non-stress conditions (Chen et al., 2002; Lannoo et al., 2007). Similar to AN3, AN4, and AN5, F-box Nictaba from $A$. thaliana and the Nictaba-like lectins from soybean were present in plant cells at low levels when grown under optimal growth conditions (no stress), suggesting that the function of the Nictaba homologs is more complex (Stefanowicz et al., 2016; Van Holle et al., 2016).

\section{The Expression of ArathNictabas Is Stress-Inducible}

Comparative analyses of the expression patterns for the different Nictaba homologs in response to stress revealed that transcript levels for the ArathNictabas are specific and vary depending on the different abiotic or biotic stress treatments performed (Figure 10).

\section{Abiotic Stress}

Arabidopsis seedlings were subjected to salt and heat stress, two major abiotic stresses. High salinity stress appears primarily as osmotic stress and as such results in the disruption of homeostasis and ion distribution in the cell (Wang et al., 2003). Heat stress results in the global inhibition of translation and secondly plants have to cope with osmotic and oxidative stress (Wang et al., 2003; Qu et al., 2013; Echevarría-Zomeño et al., 2016). The phytohormone $\mathrm{ABA}$ is a key regulator in the plant stress response to osmotic stress (Golldack et al., 2014). Moreover, ABA also plays an important role in heat stress (Vishwakarma et al., 2017).

The expression level of AN3 is generally highly upregulated after heat stress, up to sixfold, whereas ABA treatment yields a twofold upregulation of the expression level of AN3 after 5 and $10 \mathrm{~h}$ (Figures 4, 5). AN3 possibly plays a role in the heat stress response, dependent or independent of ABA (Figure 10). Taking into account that the expression level of $A N 3$ after salt treatment is not changed, AN3 most probably does not play an essential role in the ABA-dependent salt stress response.

Overall the expression level of AN4 is downregulated after ABA treatment, salt and heat stress (Figures 4, 5). Downregulation of transcript levels during stress responses is more difficult to interpret, but possibly AN4 has a negative effect on the salt and heat stress response of the plant (Figure 10). This negative effect can be due to the repression of specific steps in the stress signaling pathway. Future experiments will have to unravel if AN4 really has a negative impact on the stress responses provoked by salt and heat stress.

The expression level of AN5 shows a 1.7-fold significant upregulation after salt treatment for $10 \mathrm{~h}$ and a 2.3 -fold upregulation was observed after treatment with $A B A$ for $10 \mathrm{~h}$ (Figures 4, 5). This can be an indication that AN5 plays a role in the ABA-dependent pathway of the salt stress response (Figure 10).

\section{Biotic Stress}

Five-week-old Arabidopsis plants were subjected to different biotic stresses (Figure 6 and Supplementary Figure 2). P. syringae is a hemibiotroph that belongs to the Gram-negative plantpathogenic bacteria (Surico, 2013; Büttner, 2016). B. cinerea is a necrotrophic fungus and produces diverse phytotoxic compounds and cell-wall degrading enzymes to induce cell necrosis and as such leakage of nutrients (Mengiste, 2012). $M$. persicae is an insect belonging to the class of pierce-sucking insects and uses its stylet to feed from the phloem of the plant (Louis and Shah, 2013). Two phytohormones are known to play a major role in the defense against biotic stresses. $P$. syringae infection is activating the SA-dependent plant defense pathway while $B$. cinerea infection and $M$. persicae infestation are activating the JA-dependent plant defense pathway (Katagiri et al., 2002; Mengiste, 2012; Pieterse et al., 2012).

The expression level of AN3 shows an early 2-2.5-fold upregulation after $P$. syringae infection (after 3 days) and a twofold downregulation after 3 days of $M$. persicae infestation. The upregulation upon $P$. syringae infection correlates with the 1.5-3.5-fold upregulation of AN3 after SA treatment for 1-24 h, but does not correlate with the 2-4-fold upregulation of the expression of AN3 after 1-24 h MeJA treatment (Figures 4, 6, 10). For both hormones the expression is significantly upregulated, which is confusing because the SA- and JA-dependent defense pathways normally work antagonistically (Smith et al., 2009; Vos et al., 2013). However, it has to be noted that $P$. syringae produces coronatine, a compound which is structurally similar to JA and was shown to suppress the SA-mediated defense of the plant (Katagiri et al., 2002; Jones and Dangl, 2006). What is more, hormone cross-talk is very complex and also neutral as well as synergistic interactions between SA and JA have been reported. Timing, sequence of initiation and the relative concentration of each hormone play a role in the outcome of the SA-JA cross-talk (Vos et al., 2013).

The expression level of AN4 is upregulated two times at 5 and 7 days after $P$. syringae infection, but in general the expression level of AN4 is not changed after SA treatment, indicating that AN4 might play a role in the plant response to $P$. syringae independently of SA (Figures 4, 6, 10). Yet, only one of the three overexpression lines for AN4 shows a better tolerance than WT plants toward $P$. syringae infection (Figure 8). The better performance of transgenic line AN4_B1 can also be an off-target effect, e.g., related to the site where the T-DNA insertion in the genome took place. The significant downregulation of AN4 in the MeJA treated Arabidopsis seedlings is small and does not correlate with the expression of AN4 upon M. persicae infestation (Figures 4, 6). 
The expression level of AN5 shows an almost twofold upregulation after 3 days of $M$. persicae infestation. This is in agreement with the small upregulation of AN5 expression observed after MeJA treatment (Figures 4, 6). Possibly AN5 plays a role in the JA-dependent defense against aphids (Figure 10) (Louis and Shah, 2013). This assumption is in agreement with the repression in phloem-feeding activities of $M$. persicae as a result of overexpression of AN5 in Arabidopsis (Zhang et al., 2011). Moreover Beneteau et al. (2010) showed that recombinant AN5 at mid-range concentrations, affected weight gain of $M$. persicae nymphs. The SA signaling pathway is also known to be activated during aphid infestation, but this pathway rather facilitates aphid infestation (Louis and Shah, 2013). Transcriptome analysis of Arabidopsis subjected to the phloem-feeding insect Bemisia tabaci, the silverleaf whitefly, revealed differences in the plant responses after silverleaf whitefly and $M$. persicae infestation. Indeed, also for AN5 the response is different, since the expression of AN5 showed a twofold downregulation after B. tabaci infestation (Kempema et al., 2006). All overexpression lines for AN5 are more tolerant (though not always significant) to $P$. syringae infections compared to WT plants. Since all three independent AN5 overexpression lines show similar results and are more tolerant, this result is probably related to the overexpression of AN5 (Figure 8). At the same time, this result is surprising because the expression level of AN5 is not changed after $P$. syringae infection.

\section{Cross-talk between Abiotic and Biotic Stress}

Abiotic and biotic stresses evoke a complex cellular and molecular response in the plant to prevent damage and ensure survival of the plant. Hormones act as signaling molecules in these plant stress responses (Atkinson and Urwin, 2012; Huber and Bauerle, 2016). The phytohormones known to be important in abiotic and biotic stress responses often interact with each other. JA, SA, and ET play a role in abiotic stresses (Miura and Tada, 2014; Dar et al., 2015; Kazan, 2015; Valenzuela et al., 2016), while ABA is also important in the defense against biotic stresses (Tsuda and Katagiri, 2010; Pieterse et al., 2012; Vos et al., 2013). In addition, several growth hormones were also reported to play a role in abiotic and biotic stress, making the hormone signaling in plants subjected to stress even more complex (Tsuda and Katagiri, 2010; Kazan, 2015; Couto and Zipfel, 2016). Moreover, $\mathrm{Ca}^{2+}$ and ROS were also shown to play a role in the cross-talk between abiotic and biotic stress (Tsuda and Katagiri, 2010; Bhargava and Sawant, 2013; Couto and Zipfel, 2016; Vishwakarma et al., 2017).

Until now, most studies focused on the effect(s) of a particular stress, but our understanding of the stress signaling pathways under combinations of both abiotic and biotic stresses is still rather poor. In nature however, plants are often simultaneously exposed to multiple stresses. The presence of an abiotic stress can have a positive or a negative impact on the susceptibility to a biotic agent, and vice versa. Moreover, the plant response to multiple stresses can be totally different from the response to each of the individual ones. Therefore, it will be important to study the expression of ArathNictabas in plants subjected to a combination of stress factors to develop broad-spectrum stress tolerant crop plants in the future (Atkinson and Urwin, 2012; Rasmussen et al., 2013; Huber and Bauerle, 2016).

\section{AN4 Interacts with Two Plant Defense-Involved Enzymes}

The genome of $A$. thaliana is fully sequenced and annotated. For many proteins, interaction partners are predicted and/or experimentally proven, revealing part of the interactome of A. thaliana. Until now, no interaction partners are reported for AN4. Pull-down experiments using AN4-HIS coupled to Ni agarose beads as a bait protein and an Arabidopsis extract as prey proteins, followed by mass spectrometry revealed several possible interaction partners for AN4 (Figure 9). Two interaction partners were identified in the pull-down assays with the plant lysate originating from the salt stressed Arabidopsis seedlings. TGG1 (AT5G26000) or myrosinase 1 is a thioglucoside glucohydrolase localized in the vacuole. BGLU23 (AT3G09260) or PYK10 is a $\beta$-O-glucosidase localized in the ER bodies. A similar experiment with the plant lysate originating from non-treated Arabidopsis seedlings yielded three possible interaction partners, one which was also a significant hit in the first analysis, in particular TGG1. In addition, GDSL esterase/lipase ESM1 (AT3G14210) and protein plastid transcriptionally active 16 (PTAC16; AT3G46780) were identified as possible interaction partners (Supplementary Table 6). ESM1 is a secreted protein with a role in glucosinolate hydrolysis as a myrosinase-associated protein. PTAC16 is known as a chloroplast protein which regulates the membrane-anchoring functions of the nucleoid (Zhang et al., 2006; Burow et al., 2008; Ingelsson and Vener, 2012). It should be mentioned that the latter analysis was the result of two rather than three replicates of the soluble fraction of the non-induced E. coli cultures.

Myrosinase 1 belongs to the glycosyl hydrolase 1 family and is a $\beta$-thioglucoside glucosidase (Andersson et al., 2009). Glycosyl hydrolases hydrolyse the glycosidic bond between carbohydrates or a carbohydrate and non-carbohydrate moiety (Ahn et al., 2010). The myrosinases from A. thaliana, referred to as TGG1 - TGG6 are all grouped in the myrosinase gene family (Barth and Jander, 2006), and catalyze the hydrolysis of glucosinolates, thereby initiating the formation of isothiocyanates, nitriles, thiocyanates, epithionitriles and other reactive products (Thangstad et al., 2004; Andersson et al., 2009). Glucosinolates or thioglucosides consist of a glucose residue linked to an AA derived R-group of aliphatic, aromatic or indole types by a thioglucoside bond (Thangstad et al., 2004). In $A$. thaliana, different glucosinolates with side chains derived from methionine, tryptophan, phenylalanine and isoleucine were found (Barth and Jander, 2006). Myrosinases and glucosinolates are known to be localized in separate plant cells and consequently only make contact after tissue disruption. Myrosinases are present in the myrosin phloem idioblasts in phoem parenchyma while glucosinolates are present in the S-cells adjacent to the phloem (Thangstad et al., 2004; Barth and Jander, 2006; Andersson et al., 2009). After pathogen invasion or insect herbivory, the myrosinases can degrade glucosinolates and as such produce toxic products to protect the plant against these 
invaders. Thus, myrosinases play a role in plant defense against microbes and herbivores (Barth and Jander, 2006). Next to the myrosin phloem idioblasts, TGG1 was also reported in the guard cells (Thangstad et al., 2004).

BGLU23 or PYK10 is a $\beta$-O-glucosidase that, like myrosinase 1 , belongs to the GH1 family (Nagano et al., 2008). In total $47 \beta$-glucosidases have been identified in A. thaliana, called BGLU1 - BGLU47. BGLU23 (or PYK10) and TGG1 (or BGLU38), the two interaction partners identified for AN4, represent two of these $\beta$-glucosidases. BGLU23 is a root and hypocotyl specific $\beta$-glucosidase which is synthesized with an endoplasmic reticulum (ER) retention signal at its C-terminus (Nitz et al., 2001; Xu et al., 2004). BGLU23 is known to hydrolyze the natural substrate scopolin and other coumarin glucosides similar in structure to scopolin. Scopolin is one of the most abundant secondary metabolites in the Arabidopsis roots (Ahn et al., 2010). The resulting scopoletin is a fungitoxic compound and can be polymerized by peroxidase in the presence of $\mathrm{H}_{2} \mathrm{O}_{2}$ (Reigh et al., 1973). This can protect plant cells from the oxidative damage caused by pathogens. BGLU23 localizes to ER bodies in the roots, which are not present in rosette leaves under normal growth conditions. However, ER bodies can be induced by MeJA treatment and wounding in rosette leaves (Nagano et al., 2005; Ahn et al., 2010). Upon disruption of cells, BGLU23 forms large complexes with its binding proteins. One of these binding proteins is $\mathrm{PBP} 1$, a jacalin homolog from A. thaliana (Ahn et al., 2010). BGLU23 is a glycoprotein with three high-mannose oligosaccharides which can be recognized by PBP1, the jacalin homolog. It is possible that PBP1 participates in the BGLU23 and ER body-mediated defense systems against herbivores and pathogens (Matsushima et al., 2004). PBP1 is thought to act as a molecular chaperone that helps the correct polymerization of BGLU23 when tissues are damaged. This polymerization of BGLU23 is necessary for its activity (Nagano et al., 2005, 2008).

At first sight, there is no obvious link between AN4 and the plant defense-involved enzymes TGG1 and BGLU23. However, interesting observations were made when the localization, the expression pattern and the expression levels were compared in more detail. Protein localization and expression under normal growth conditions can be compared at subcellular level, according to cell type and at tissue level. AN4 resides in the nucleus and the cytoplasm of the cell and its expression under normal growth conditions is significantly higher in the roots compared to 6-day-old seedlings (Figures 2, 3). Jakoby et al. (2008) identified AN4 as one of the 5\% most highly expressed genes in mature Arabidopsis trichomes. Moreover, they reported a strong enrichment of genes involved in root atrichoblast differentiation in the trichome transcriptome (Jakoby et al., 2008). The sequence encoding TGG1 contains a signal peptide and the protein localizes to the vacuole (Thangstad et al., 2004). Xue et al. (1995) reported the expression of TGG1 in the leaves, stems and floral organs. At cell level, TGG1 was especially found in the guard cells and in phloem myrosin cells. Myrosin cells or idioblasts are specific cells that differ greatly from the neighboring cells in size, structure and content. The morphology of these specific cells can vary in different organs, tissues and developmental stages (Thangstad et al., 2004). BGLU23, like TGG1, is synthesized with a signal peptide, but also contains an ER-retention signal (KDEL sequence). This $\beta$-O-glucosidase, also called PYK10 is specifically localized in ER bodies and is root specific (Nitz et al., 2001; Matsushima et al., 2003; Ahn et al., 2010). BGLU23 was also reported in the Arabidopsis plasmodesmal proteome, but probably represents a cytoplasmic contaminant in this fraction (Fernandez-Calvino et al., 2011).

A striking similarity between AN4 and BGLU23 at tissue level is their expression in the roots of Arabidopsis. However, according to microarray data (eFP browser) the expression level of BGLU23 in the roots is much higher than the expression level of AN4 under normal growth conditions (Winter et al., 2007). Furthermore, it is interesting to note that BGLU23 was also identified as one of the $5 \%$ most highly expressed genes in mature Arabidopsis trichomes (Jakoby et al., 2008). Although trichomes are present on the leaves, it is reported that trichome development in leaves and atrichoblast development in roots share a network of transcription factors (Pesch and Hülskamp, 2004; Schellmann et al., 2007). However, it is not known whether trichomes and atrichoblasts really share common patterns of gene expression (Jakoby et al., 2008). Jakoby et al. (2008) reported high activity of genes involved in the glucosinolate pathways in trichomes, indicating the role of trichomes in plant defense. Possibly AN4 and BGLU23 can interact in the roots or trichomes when the plant is subjected to stress. To do so, at least one of the two proteins would have to change its subcellular localization. Changes in subcellular localization after stress have been reported before (García et al., 2010; Moore et al., 2011). Alternatively AN4 and BGLU23 can interact as a result of the cell damage provoked by, e.g., pathogens.

Another similarity at tissue level is seen between TGG1 and AN4. TGG1 was especially abundant in the phloem myrosin cells whereas AN4 was reported as a PP2 of Arabidopsis. PP2 proteins are most abundant in the phloem sap. Though the sequence of AN4 shows high similarity to the PP2 domain, a protein domain that is conserved among many species in the plant kingdom, there is no evidence for the presence of AN4 in the phloem, except for the microarray data from eFP browser (Dinant et al., 2003; Thangstad et al., 2004; Winter et al., 2007).

The expression level of AN4 is low under normal growth conditions and its expression remains low or is even downregulated after all investigated stress treatments, except for the P. syringae infection. P. syringae infection of 5-week-old rosette leaves resulted in approximately two times upregulation of AN4 transcript levels at the latest timepoints (after 5 and 7 days). According to the microarray data of the eFP browser, BGLU23 shows a sixfold upregulation $24 \mathrm{~h}$ after $P$. syringae infection, but TGG1 shows a small downregulation (Winter et al., 2007). It has to be mentioned that our data also first show a twofold significant downregulation of AN4 about $24 \mathrm{~h}$ after P. syringae infection. Although TGG1 and AN4 show a similar regulation $24 \mathrm{~h}$ after $P$. syringae infection, their absolute expression levels are very different, TGG1 is present in much higher levels in infected Arabidopsis leaves than AN4 (Figure 10). 
At present, it remains unclear if the expression of BGLU23 and $A N 4$, and their interaction in the trichomes is part of the defense response against Pseudomonas.

Since the pull-down analysis is an in vitro analysis and the plant extract contains a mixture of proteins derived from different cell compartments, it is uncertain whether the interaction partners retrieved from the pull-down assay represent true in vivo interaction partners for AN4. Additionally, TGG1 and BGLU23 are two proteins that are quite abundant in Arabidopsis seedlings, whereas AN4 is expressed at a much lower level. Future experiments are thus necessary to confirm these protein interactions in vivo.

To conclude, similar to Nictaba from tobacco the ArathNictabas under study are also localized to the nucleus and/or the cytoplasm (Chen et al., 2002). ArathNictaba 3-5 are expressed at very low levels in all tissues during the development of A. thaliana under normal growth conditions, but their expression is clearly stress-inducible, though specific stresses trigger differential expression of ArathNictaba 3-5. Thus, it can be concluded that expression patterns for the ArathNictabas are specific and vary for different abiotic or biotic stress treatments (Figure 10). Our data suggest that ArathNictabas could play a role in the stress response of $A$. thaliana. Future research is needed to investigate the subcellular localization of the ArathNictabas after plants that have been exposed to stress situations. In addition, analyses with mutant lines can help to decipher the role of the ArathNictabas in the plant stress response. Finally, the interaction of AN4 with TGG1 and BGLU23 has to be confirmed in vivo and it has to be shown whether this binding is a protein-protein or a protein-carbohydrate interaction.

\section{REFERENCES}

Ahn, Y. O., Shimizu, B., Sakata, K., Gantulga, D., Zhou, C., Zhou, Z., et al. (2010). Scopolin-hydrolyzing $\beta$-glucosidases in roots of Arabidopsis. Plant Cell Physiol. 51, 132-143. doi: 10.1093/pcp/pcp174

Andersson, D., Chakrabarty, R., Bejai, S., Zhang, J., Rask, L., and Meijer, J. (2009). Myrosinases from root and leaves of Arabidopsis thaliana have different catalytic properties. Phytochemistry 70, 1345-1354. doi: 10.1016/j.phytochem. 2009.07.036

Atkinson, N. J., and Urwin, P. E. (2012). The interaction of plant biotic and abiotic stresses: from genes to the field. J. Exp. Bot. 63, 3523-3544. doi: 10.1093/jxb/ ers 100

Audenaert, K., De Meyer, G. B., and Höfte, M. M. (2002). Abscisic acid determines basal susceptibility of tomato to Botrytis cinerea and suppresses salicylic aciddependent signaling mechanisms. Plant Physiol. 128, 491-501. doi: 10.1104/pp. 010605

Barth, C., and Jander, G. (2006). Arabidopsis myrosinases TGG1 and TGG2 have redundant function in glucosinolate breakdown and insect defense. Plant J. 46, 549-562. doi: 10.1111/j.1365-313X.2006.02716.x

Beneteau, J., Renard, D., Marché, L., Douville, E., Lavenant, L., Rahbé, Y., et al. (2010). Binding properties of the $N$-acetylglucosamine and high-mannose $N$-glycan PP2-A1 phloem lectin in Arabidopsis. Plant Physiol. 153, 1345-1361. doi: $10.1104 / \mathrm{pp} .110 .153882$

Bhargava, S., and Sawant, K. (2013). Drought stress adaptation: metabolic adjustment and regulation of gene expression. Plant Breed. 132, 21-32. doi: 10.1111/Pbr.12004

Brameier, M., Krings, A., and MacCallum, R. M. (2007). NucPred - Predicting nuclear localization of proteins. Bioinformatics 23, 1159-1160. doi: 10.1093/ bioinformatics/btm066

\section{AUTHOR CONTRIBUTIONS}

LE and EVD outlined and designed the study. LE performed the experiments, analyzed and interpreted the data and prepared the manuscript. KS provided some cDNAs of stress experiments and helped with the $P$. syringae stress experiment on WT plants. EVD conceived and supervised the experiments and critically revised the manuscript. All authors have read, revised, and approved the final manuscript.

\section{FUNDING}

This work was supported by the Research Council of Ghent University [project 01G00515].

\section{ACKNOWLEDGMENTS}

The authors wish to thank Dr. Na Yu and Dr. Mohammed Hamshou for their advice with the setup and analysis of the aphid experiments. They wish to thank the VIB Proteomics Expertise Center (Center for Medical Biotechnology, VIB, UGhent) for the mass spectrometry.

\section{SUPPLEMENTARY MATERIAL}

The Supplementary Material for this article can be found online at: https://www.frontiersin.org/articles/10.3389/fpls.2017.02218/ full\#supplementary-material

Brouwer, M., Lievens, B., Van Hemelrijck, W., Van Den Ackerveken, G., Cammue, B. P. A., and Thomma, B. P. H. J. (2003). Quantification of disease progression of several microbial pathogens on Arabidopsis thaliana using realtime fluorescence PCR. FEMS Microbiol. Lett. 228, 241-248. doi: 10.1016/ S0378-1097(03)00759-6

Burow, M., Zhang, Z. Y., Ober, J. A., Lambrix, V. M., Wittstock, U., Gershenzon, J., et al. (2008). ESP and ESM1 mediate indol-3-acetonitrile production from indol-3-ylmethyl glucosinolate in Arabidopsis. Phytochemistry 69, 663-671. doi: $10.1016 /$ j.phytochem.2007.08.027

Büttner, D. (2016). Behind the lines-actions of bacterial type III effector proteins in plant cells. FEMS Microbiol. Rev. 40, 894-937. doi: 10.1093/femsre/ fuw026

Cayla, T., Batailler, B., Le Hir, R., Revers, F., Anstead, J. A., Thompson, G. A., et al. (2015). Live imaging of companion cells and sieve elements in Arabidopsis leaves. PLOS ONE 10:e0118122. doi: 10.1371/journal.pone.011 8122

Chen, Y., Peumans, W., Hause, B., Bras, J., Kumar, M., Proost, P., et al. (2002). Jasmonate methyl ester induces the synthesis of a cytoplasmic/nuclear chitooligosaccharide-binding lectin in tobacco leaves. FASEB J. 16, 905-907. doi: 10.1096/fj.01-0598fje

Clough, S. J., and Bent, A. (1998). Floral dip: a simplified method for Agrobacterium mediated transformation of Arabidopsis thaliana. Plant J. 16, 735-743. doi: 10.1046/j.1365-313x.1998.00343.x

Couto, D., and Zipfel, C. (2016). Regulation of pattern recognition receptor signalling in plants. Nat. Rev. Immunol. 16, 537-552. doi: 10.1038/nri.2016.77

Czechowski, T., Stitt, M., Altmann, T., Udvardi, M. K., and Scheible, W.-R. (2005). Genome-wide identification and testing of superior reference genes for transcript normalization in Arabidopsis. Plant Physiol. 139, 5-17. doi: 10.1104/ pp.105.063743 
Dar, T. A., Uddin, M., Khan, M. M. A., Hakeem, K. R., and Jaleel, H. (2015). Jasmonates counter plant stress: a review. Environ. Exp. Bot. 115, 49-57. doi: 10.1016/j.envexpbot.2015.02.010

Delporte, A., De Vos, W. H., and Van Damme, E. J. M. (2014). In vivo interaction between the tobacco lectin and the core histone proteins. J. Plant Physiol. 171, 1149-1156. doi: 10.1016/j.jplph.2014.04.008

Delporte, A., Van Holle, S., Lannoo, N., and Van Damme, E. J. M. (2015). The tobacco lectin, prototype of the family of Nictaba-related proteins. Curr. Protein Pept. Sci. 16, 5-16.

Delporte, A., Van Holle, S., and Van Damme, E. J. M. (2013). Qualitative and quantitative analysis of the Nictaba promoter activity during development in Nicotiana tabacum. Plant Physiol. Biochem. 67, 162-168. doi: 10.1016/j.plaphy. 2013.03.005

Dinant, S., Clark, A. M., Zhu, Y., Vilaine, F., Palauqui, J.-C., Kusiak, C., et al. (2003). Diversity of the superfamily of phloem lectins (phloem protein 2) in angiosperms. Plant Physiol. 131, 114-128. doi: 10.1104/pp. 013086

Echevarría-Zomeño, S., Fernández-Calvino, L., Castro-Sanz, A. B., López, J. A., Vázquez, J., and Castellano, M. M. (2016). Dissecting the proteome dynamics of the early heat stress response leading to plant survival or death in Arabidopsis. Plant Cell Environ. 39, 1264-1278. doi: 10.1111/pce.12664

Eggermont, L., Verstraeten, B., and Van Damme, E. J. M. (2017). Genomewide screening for lectin motifs in Arabidopsis thaliana. Plant Genome 10, doi: 10.3835/plantgenome2017.02.0010

Fernandez-Calvino, L., Faulkner, C., Walshaw, J., Saalbach, G., Bayer, E., BenitezAlfonso, Y., et al. (2011). Arabidopsis plasmodesmal proteome. PLOS ONE 6:e18880. doi: 10.1371/journal.pone.0018880

García, A. V., Blanvillain-Baufumé, S., Huibers, R., Wiermer, M., Li, G., Gobbato, E., et al. (2010). Balanced nuclear and cytoplasmic activities of EDS1 are required for a complete plant innate immune response. PLOS Pathog. 6:e1000970. doi: 10.1371/journal.ppat.1000970

Gibson, D. G., Young, L., Chuang, R.-Y., Venter, J. C., Hutchison, C. A., and Smith, H. O. (2009). Enzymatic assembly of DNA molecules up to several hundred kilobases. Nat. Methods 6, 343-345. doi: 10.1038/nmeth. 1318

Golldack, D., Li, C., Mohan, H., and Probst, N. (2014). Tolerance to drought and salt stress in plants: unraveling the signaling networks. Front. Plant Sci. 5:151. doi: $10.3389 /$ fpls. 2014.00151

Görlich, D. (1998). Transport into and out of the cell nucleus. EMBO J. 17, 2721-2727. doi: 10.1093/emboj/17.10.2721

Harrison, S. J., Mott, E. K., Parsley, K., Aspinall, S., Gray, J. C., and Cottage, A. (2006). A rapid and robust method of identifying transformed Arabidopsis thaliana seedlings following floral dip transformation. Plant Methods 2:19. doi: 10.1186/1746-4811-2-19

Hellemans, J., Mortier, G., De Paepe, A., Speleman, F., and Vandesompele, J. (2007). qBase relative quantification framework and software for management and automated analysis of real-time quantitative PCR data. Genome Biol. 8:19. doi: 10.1186/gb-2007-8-2-r19

Hooper, C. M., Tanz, S. K., Castleden, I. R., Vacher, M. A., Small, I. D., and Millar, A. H. (2014). SUBAcon: a consensus algorithm for unifying the subcellular localization data of the Arabidopsis proteome. Bioinformatics 30, 3356-3364. doi: 10.1093/bioinformatics/btu550

Hruz, T., Laule, O., Szabo, G., Wessendorp, F., Bleuler, S., Oertle, L., et al. (2008). Genevestigator v3: a reference expression database for the metaanalysis of transcriptomes. Adv. Bioinformatics 2008:420747. doi: 10.1155/2008/ 420747

Huber, A. E., and Bauerle, T. L. (2016). Long-distance plant signaling pathways in response to multiple stressors: the gap in knowledge. J. Exp. Bot. 67, 2063-2079. doi: 10.1093/jxb/erw099

Ingelsson, B., and Vener, A. V. (2012). Phosphoproteomics of Arabidopsis chloroplasts reveals involvement of the STN7 kinase in phosphorylation of nucleoid protein PTAC16. FEBS Lett. 586, 1265-1271. doi: 10.1016/j.febslet. 2012.03.061

Jakoby, M. J., Falkenhan, D., Mader, M. T., Brininstool, G., Wischnitzki, E., Platz, N., et al. (2008). Transcriptional profiling of mature Arabidopsis trichomes reveals that NOECK encodes the MIXTA-like transcriptional regulator MYB106. Plant Physiol. 148, 1583-1602. doi: 10.1104/pp.108. 126979
Jones, J. D. G., and Dangl, J. L. (2006). The plant immune system. Nature 444, 323-329. doi: 10.1038/nature05286

Käll, L., Krogh, A., and Sonnhammer, E. L. L. (2004). A combined transmembrane topology and signal peptide prediction method. J. Mol. Biol. 338, 1027-1036. doi: 10.1016/j.jmb.2004.03.016

Karimi, M., Inzé, D., and Depicker, A. (2002). GATEWAYTM vectors for Agrobacterium-mediated plant transformation. Trends Plant Sci. 7, 193-195. doi: 10.1016/S1360-1385(02)02251-3

Katagiri, F., Thilmony, R., and He, S. Y. (2002). The Arabidopsis thalianaPseudomonas syringae interaction. Arabidopsis Book 1:e0039. doi: 10.1199/tab. 0039

Kazan, K. (2015). Diverse roles of jasmonates and ethylene in abiotic stress tolerance. Trends Plant Sci. 20, 219-229. doi: 10.1016/j.tplants.2015. 02.001

Kempema, L. A., Cui, X., Holzer, F. M., and Walling, L. L. (2006). Arabidopsis transcriptome changes in response to phloem-feeding silverleaf whitefly nymphs. Similarities and distinctions in responses to aphids. Plant Physiol. 143, 849-865. doi: 10.1104/pp.106.090662

Kosugi, S., Hasebe, M., Matsumura, N., Takashima, H., Miyamoto-Sato, E., Tomita, M., et al. (2009). Six classes of nuclear localization signals specific to different binding grooves of importin $\alpha$. J. Biol. Chem. 284, 478-485. doi: 10.1074/jbc.M807017200

Laemmli, U. K. (1970). Cleavage of structural proteins during the assembly of the head of bacteriophage T4. Nature 227, 680-685. doi: 10.1038/227 $680 \mathrm{a} 0$

Lange, A., Mills, R. E., Lange, C. J., Stewart, M., Devine, S. E., and Corbett, A. H. (2007). Classical nuclear localization signals: definition, function, and interaction with importin $\alpha$. J. Biol. Chem. 282, 5101-5105. doi: 10.1074/jbc. R600026200

Lannoo, N., Peumans, W. J., Van Pamel, E., Alvarez, R., Xiong, T. C., Hause, G., et al. (2006). Localization and in vitro binding studies suggest that the cytoplasmic/nuclear tobacco lectin can interact in situ with high-mannose and complex N-glycans. FEBS Lett. 580, 6329-6337. doi: 10.1016/j.febslet.2006.10.044

Lannoo, N., and Van Damme, E. J. M. (2010). Nucleocytoplasmic plant lectins. Biochim. Biophys. Acta 1800, 190-201. doi: 10.1016/j.bbagen.2009. 07.021

Lannoo, N., and Van Damme, E. J. M. (2014). Lectin domains at the frontiers of plant defense. Front. Plant Sci. 5:397. doi: 10.3389/fpls.2014. 00397

Lannoo, N., Vandenborre, G., Miersch, O., Smagghe, G., Wasternack, C., Peumans, W. J., et al. (2007). The jasmonate-induced expression of the Nicotiana tabacum leaf lectin. Plant Cell Physiol. 48, 1207-1218. doi: 10.1093/pcp/ pcm090

Louis, J., and Shah, J. (2013). Arabidopsis thaliana-Myzus persicae interaction: shaping the understanding of plant defense against phloem-feeding aphids. Front. Plant Sci. 4:213. doi: 10.3389/fpls.2013.00213

Matsushima, R., Fukao, Y., Nishimura, M., and Hara-Nishimura, I. (2004). NAI1 gene encodes a basic-helix-loop-helix-type putative transcription factor that regulates the formation of an endoplasmic reticulum-derived structure, the ER body. Plant Cell 16, 1536-1549. doi: 10.1105/tpc.021154

Matsushima, R., Kondo, M., Nishimura, M., and Hara-Nishimura, I. (2003). A novel ER-derived compartment, the ER body, selectively accumulates a $\beta$-glucosidase with an ER-retention signal in Arabidopsis. Plant J. 33, 493-502. doi: 10.1046/j.1365-313X.2003.01636.x

Mengiste, T. (2012). Plant immunity to necrotrophs. Annu. Rev. Phytopathol. 50, 267-294. doi: 10.1146/annurev-phyto-081211-172955

Miura, K., and Tada, Y. (2014). Regulation of water, salinity, and cold stress responses by salicylic acid. Front. Plant Sci. 5:4. doi: 10.3389/fpls.2014. 00004

Moore, J. W., Loake, G. J., and Spoel, S. H. (2011). Transcription dynamics in plant immunity. Plant Cell 23, 2809-2820. doi: 10.1105/tpc.111.087346

Nagano, A. J., Fukao, Y., Fujiwara, M., Nishimura, M., and Hara-Nishimura, I. (2008). Antagonistic jacalin-related lectins regulate the size of ER bodytype $\beta$-glucosidase complexes in Arabidopsis thaliana. Plant Cell Physiol. 49, 969-980. doi: 10.1093/pcp/pcn075

Nagano, A. J., Matsushima, R., and Hara-Nishimura, I. (2005). Activation of an ERbody-localized $\beta$-glucosidase via a cytosolic binding partner in damaged tissues 
of Arabidopsis thaliana. Plant Cell Physiol. 46, 1140-1148. doi: 10.1093/pcp/ pci126

Nitz, I., Berkefeld, H., Puzio, P. S., and Grundler, F. M. W. (2001). Pyk10, a seedling and root specific gene and promoter from Arabidopsis thaliana. Plant Sci. 161, 337-346. doi: 10.1016/S0168-9452(01)00 412-5

Paine, P. L., Moore, L. C., and Horowitz, S. B. (1975). Nuclear envelope permeability. Nature 254, 109-114. doi: 10.1038/254109a0

Pemberton, L. F., and Paschal, B. M. (2005). Mechanisms of receptor-mediated nuclear import and nuclear export. Traffic 6, 187-198. doi: 10.1111/j.1600-0854. 2005.00270.x

Pesch, M., and Hülskamp, M. (2004). Creating a two-dimensional pattern de novo during Arabidopsis trichome and root hair initiation. Curr. Opin. Genet. Dev. 14, 422-427. doi: 10.1016/j.gde.2004.06.007

Petersen, T. N., Brunak, S., von Heijne, G., and Nielsen, H. (2011). SignalP 4.0: discriminating signal peptides from transmembrane regions. Nat. Methods 8 , 785-786. doi: 10.1038/nmeth.1701

Peumans, W. J., and Van Damme, E. J. M. (1995). Lectins as plant defense proteins. Plant Physiol. 109, 347-352. doi: 10.1104/pp.109.2.347

Pfaffl, M. W., Horgan, G. W., and Dempfle, L. (2002). Relative expression software tool (REST) for group-wise comparison and statistical analysis of relative expression results in real-time PCR. Nucleic Acids Res. 30:e36. doi: 10.1093/nar/ 30.9.e36

Pieterse, C. M. J., Van der Does, D., Zamioudis, C., Leon-Reyes, A., and Van Wees, S. C. M. (2012). Hormonal modulation of plant immunity. Annu. Rev. Cell Dev. Biol. 28, 489-521. doi: 10.1146/annurev-cellbio-092910154055

Pieterse, C. M. J., van Wees, S. C., Hoffland, E., van Pelt, J. A., and van Loon, L. C. (1996). Systemic resistance in Arabidopsis induced by biocontrol bacteria is independent of salicylic acid accumulation and pathogenesisrelated gene expression. Plant Cell 8, 1225-1237. doi: 10.1105/tpc.8.8. 1225

Qu, A.-L., Ding, Y.-F., Jiang, Q., and Zhu, C. (2013). Molecular mechanisms of the plant heat stress response. Biochem. Biophys. Res. Commun. 432, 203-207. doi: 10.1016/j.bbrc.2013.01.104

Rasmussen, S., Barah, P., Suarez-Rodriguez, M. C., Bressendorff, S., Friis, P., Costantino, P., et al. (2013). Transcriptome responses to combinations of stresses in Arabidopsis. Plant Physiol. 161, 1783-1794. doi: 10.1104/pp.112. 210773

Reigh, D. L., Wender, S. H., and Smith, E. C. (1973). Scopoletin: a substrate for an isoperoxidase from Nicotiana tabacum tissue culture W-38. Phytochemistry 12, 1265-1268. doi: 10.1016/0031-9422(73)80550-3

Schellmann, S., Hülskamp, M., and Uhrig, J. (2007). Epidermal pattern formation in the root and shoot of Arabidopsis. Biochem. Soc. Trans. 35, 146-148. doi: 10.1042/BST0350146

Schindelin, J., Arganda-Carreras, I., Frise, E., Kaynig, V., Longair, M., Pietzsch, T., et al. (2012). Fiji: an open source platform for biological image analysis. Nat. Methods 9, 676-682. doi: 10.1038/nmeth.2019.Fiji

Schouppe, D., Ghesquière, B., Menschaert, G., De Vos, W. H., Bourque, S., Trooskens, G., et al. (2011). Interaction of the tobacco lectin with histone proteins. Plant Physiol. 155, 1091-1102. doi: 10.1104/pp.110. 170134

Schouppe, D., Rougé, P., Lasanajak, Y., Barre, A., Smith, D. F., Proost, P., et al. (2010). Mutational analysis of the carbohydrate binding activity of the tobacco lectin. Glycoconj. J. 27, 613-623. doi: 10.1007/s10719-0109305-2

Shahidi-Noghabi, S., Van Damme, E. J. M., and Smagghe, G. (2009). Expression of Sambucus nigra agglutinin (SNA-I) from elderberry bark in transgenic tobacco plants results in enhanced resistance to different insect species. Trans. Res. 18, 249-259. doi: 10.1007/s11248-008-9215-2

Smith, J. L., De Moraes, C. M., and Mescher, M. C. (2009). Jasmonate- and salicylate-mediated plant defense responses to insect herbivores, pathogens and parasitic plants. Pest Manag. Sci. 65, 497-503. doi: 10.1002/ps. 1714

Sparkes, I. A., Runions, J., Kearns, A., and Hawes, C. (2006). Rapid, transient expression of fluorescent fusion proteins in tobacco plants and generation of stably transformed plants. Nat. Protoc. 1, 2019-2025. doi: 10.1038/nprot. 2006.286
Stefanowicz, K., Lannoo, N., Proost, P., and Van Damme, E. J. M. (2012). Arabidopsis F-box protein containing a Nictaba-related lectin domain interacts with $N$-acetyllactosamine structures. FEBS Open Bio 2, 151-158. doi: 10.1016/j. fob.2012.06.002

Stefanowicz, K., Lannoo, N., Zhao, Y., Eggermont, L., Van Hove, J., Al Atalah, B., et al. (2016). Glycan-binding F-box protein from Arabidopsis thaliana protects plants from Pseudomonas syringae infection. BMC Plant Biol. 16:213. doi: 10.1186/s12870-016-0905-2

Suh, E. K., and Gumbiner, B. M. (2003). Translocation of $\beta$-catenin into the nucleus independent of interactions with FG-rich nucleoporins. Exp. Cell Res. 290, 447-456. doi: 10.1016/S0014-4827(03)00 370-7

Surico, G. (2013). The concepts of plant pathogenicity, virulence/avirulence and effector proteins by a teacher of plant pathology. Phytopathol. Mediterr. 52, 399-417. doi: 10.14601/Phytopathol_Mediterr-12077

Thangstad, O. P., Gilde, B., Chadchawan, S., Seem, M., Husebye, H., Bradley, D., et al. (2004). Cell specific, cross-species expression of myrosinases in Brassica napus, Arabidopsis thaliana and Nicotiana tabacum. Plant Mol. Biol. 54, 597-611. doi: 10.1023/B:PLAN.0000038272.99590.10

Tsuda, K., and Katagiri, F. (2010). Comparing signaling mechanisms engaged in pattern-triggered and effector-triggered immunity. Curr. Opin. Plant Biol. 13, 459-465. doi: 10.1016/j.pbi.2010.04.006

Valenzuela, C. E., Acevedo-Acevedo, O., Miranda, G. S., Vergara-Barros, P., Holuigue, L., Figueroa, C. R., et al. (2016). Salt stress response triggers activation of the jasmonate signaling pathway leading to inhibition of cell elongation in Arabidopsis primary root. J. Exp. Bot. 67, 4209-4220. doi: 10.1093/jxb/ erw202

Van Damme, E. J. M., Lannoo, N., and Peumans, W. J. (2008). Plant lectins. Adv. Bot. Res. 48, 107-209. doi: 10.1016/S0065-2296(08)00 403-5

Van Holle, S., Rougé, P., and Van Damme, E. J. M. (2017). Evolution and structural diversification of Nictaba-like lectin genes in food crops with a focus on soybean (Glycine max). Ann. Bot. 119, 901-914. doi: 10.1093/aob/ mcw259

Van Holle, S., Smagghe, G., and Van Damme, E. J. M. (2016). Overexpression of Nictaba-like lectin genes from Glycine max confers tolerance toward Pseudomonas syringae infection, aphid infestation and salt stress in transgenic Arabidopsis plants. Front. Plant Sci. 7:1590. doi: 10.3389/fpls.2016. 01590

Vandenborre, G., Van Damme, E. J. M., and Smagghe, G. (2009). Nicotiana tabacum agglutinin expression in response to different biotic challengers. Arthropod. Plant. Interact. 3, 193-202. doi: 10.1007/s11829-0099075-6

Vishwakarma, K., Upadhyay, N., Kumar, N., Yadav, G., Singh, J., Mishra, R., et al. (2017). Abscisic acid signaling and abiotic stress tolerance in plants: a review on current knowledge and future prospects. Front. Plant Sci. 8:161. doi: 10.3389/FPLS.2017.00161

Vos, I. A., Pieterse, C. M. J., and Van Wees, S. C. M. (2013). Costs and benefits of hormone-regulated plant defences. Plant Pathol. 62, 43-55. doi: 10.1111/ppa. 12105

Wang, R., and Brattain, M. G. (2007). The maximal size of protein to diffuse through the nuclear pore is larger than $60 \mathrm{kDa}$. FEBS Lett. 581, 3164-3170. doi: 10.1016/j.febslet.2007.05.082

Wang, W., Vinocur, B., and Altman, A. (2003). Plant responses to drought, salinity and extreme temperatures: towards genetic engineering for stress tolerance. Planta 218, 1-14. doi: 10.1007/s00425-003-1 105-5

Winter, D., Vinegar, B., Nahal, H., Ammar, R., Wilson, G. V., and Provart, N. J. (2007). An "electronic fluorescent pictograph" browser for exploring and analyzing large-scale biological data sets. PLOS ONE 2:e718. doi: 10.1371/ journal.pone.0000718

Xu, Z., Escamilla-Treviño, L., Zeng, L., Lalgondar, M., Bevan, D., Winkel, B., et al. (2004). Functional genomic analysis of Arabidopsis thaliana glycoside hydrolase family 1. Plant Mol. Biol. 55, 343-367. doi: 10.1007/s11103-0040790-1

Xue, J., Jørgensen, M., Pihlgren, U., and Rask, L. (1995). The myrosinase gene family in Arabidopsis thaliana: gene organization, expression and evolution. Plant Mol. Biol. 27, 911-922. 
Zhang, C., Shi, H., Chen, L., Wang, X., Lü, B., Zhang, S., et al. (2011). Harpininduced expression and transgenic overexpression of the phloem protein gene AtPP2-A1 in Arabidopsis repress phloem feeding of the green peach aphid Myzus persicae. BMC Plant Biol. 11:11. doi: 10.1186/1471-2229-11-11

Zhang, Z., Ober, J. A., and Kliebenstein, D. J. (2006). The gene controlling the quantitative trait locus EPITHIOSPECIFIER MODIFIER1 alters glucosinolate hydrolysis and insect resistance in Arabidopsis. Plant Cell 18, 1524-1536. doi: $10.1105 /$ tpc. 105.039602

Ziemienowicz, A., Haasen, D., Staiger, D., and Merkle, T. (2003). Arabidopsis transportin1 is the nuclear import receptor for the circadian clock-regulated RNA-binding protein AtGRP7. Plant Mol. Biol. 53, 201-212. doi: 10.1023/B: PLAN.0000009288.46713.1f
Conflict of Interest Statement: The authors declare that the research was conducted in the absence of any commercial or financial relationships that could be construed as a potential conflict of interest.

The reviewer CF and handling editor declared their shared affiliation.

Copyright $\odot 2018$ Eggermont, Stefanowicz and Van Damme. This is an open-access article distributed under the terms of the Creative Commons Attribution License (CC BY). The use, distribution or reproduction in other forums is permitted, provided the original author(s) or licensor are credited and that the original publication in this journal is cited, in accordance with accepted academic practice. No use, distribution or reproduction is permitted which does not comply with these terms. 